\title{
The dust SED of dwarf galaxies
}

\section{The case of NGC $4214^{\star}$}

\author{
I. Hermelo ${ }^{1}$, U. Lisenfeld ${ }^{1}$, M. Relaño ${ }^{1}$, R. J. Tuffs ${ }^{2}$, C. C. Popescu ${ }^{3}$, and B. Groves ${ }^{4}$ \\ 1 Departamento de Física Teórica y del Cosmos, Universidad de Granada, Spain \\ e-mail: [israelhermelo;ute;mrelano]@ugr.es \\ 2 Max-Planck-Institut für Kernphysik, Saupfercheckweg 1, 69117 Heidelberg, Germany \\ 3 Jeremiah Horrocks Institute for Astrophysics and Supercomputing, University of Central Lancashire, PR1 2HE, Preston, UK \\ 4 Max-Planck-Institut für Astronomie, Königstuhl 17, 69117 Heidelberg, Germany
}

Received 6 July 2012 / Accepted 16 October 2012

\begin{abstract}
Context. High-resolution data from Spitzer, Herschel, and Planck allow us to probe the entire spectral energy distribution (SED) of morphologically separated components of the dust emission from nearby galaxies and allow a more detailed comparison between data and models.

Aims. We wish to establish the physical origin of dust heating and emission based on radiation transfer models, that self-consistently connect the emission components from diffuse dust and the dust in massive star forming regions.

Methods. NGC 4214 is a nearby dwarf galaxy with a large set of ancillary data, ranging from the ultraviolet (UV) to radio, including maps from Spitzer and Herschel and detections from Planck. We mapped this galaxy with MAMBO at $1.2 \mathrm{~mm}$ at the IRAM $30 \mathrm{~m}$ telescope. We extracted separate dust emission components for the HII regions (plus their associated PDRs on pc scales) and for the diffuse dust (on kpc scales). We analysed the full UV to FIR/submm SED of the galaxy using a radiation transfer model that self-consistently treats the dust emission from diffuse and star forming (SF) complexes components, considering the illumination of diffuse dust both by the distributed stellar populations and by escaping light from the HII regions. While maintaining consistency within the framework of this model, we additionally used a model that provides a detailed description of the dust emission from the HII regions and their surrounding PDRs on pc scales. Thanks to the large amount of available data and many previous studies for NGC 4214, very few free parameters remained in the model fitting process.

Results. We achieve a satisfactory fit for the emission from HII + PDR regions on pc scales, with the exception of the emission at $8 \mu \mathrm{m}$, which is underpredicted by the model. For the diffuse emission we achieve a good fit if we assume that about 40-65\% of the emission escaping the HII + PDR regions is able to leave the galaxy without passing through a diffuse ISM, which is not an unlikely scenario for a dwarf galaxy that has recently undergone a nuclear starburst. We determine a dust-to-gas mass ratio of 350-470, which is close to the expected value based on the metallicity.
\end{abstract}

Key words. dust, extinction - galaxies: individual: NGC 4214 - galaxies: dwarf - galaxies: ISM - galaxies: star formation submillimeter: galaxies

\section{Introduction}

Interstellar dust is a component of the interstellar medium (ISM) that is present in all phases from dense molecular clouds to the warm ionized regions around massive stars. It plays an important role in star formation (SF) and in the overall energy budget of a galaxy. Measurements of the dust re-emission from galaxies is a powerful technique to quantify the SF. The details, however, are not fully understood yet because the emission depends on several parameters as the geometry, the mixture between dust, stars and HII regions, and also on the dust properties in the ISM, which are expected to vary as a function of metallicity and are known to depend on the environment.

The study of dust properties (e.g. mass, extinction coefficient) has been the subject of considerable debate. Much progress has been made in recent years thanks to data from the satellites Spitzer, Herschel, and Planck, which allow to probe the entire wavelength range of the spectral energy distribution

* FITS files of all the images are only available at the CDS via anonymous ftp to cdsarc.u-strasbg. fr (130.79.128.5) or via http://cdsarc.u-strasbg.fr/viz-bin/qcat?]/A+A/549/A70
(SED) of the dust emission for the first time, from the midinfrared (MIR) to the submillimetre (submm), for a large number of galaxies at the best resolution ever.

The dust SED is a key observation for understanding the properties of dust. The dust SED of dwarf galaxies frequently shows differences to those of spiral galaxies. The main differences are the following. (i) The SED of dwarf galaxies shows a relatively low emission at $8 \mu \mathrm{m}$, most likely due to a relatively lower content of polycyclic aromatic hydrocarbons (PAHs) at low metallicities (e.g. Draine et al. 2007; Galliano et al. 2008; Engelbracht et al. 2008). (ii) A submm "excess" has been found in the SED of many, mostly actively star-forming, lowmetallicity galaxies (Lisenfeld et al. 2002; Galliano et al. 2003, 2005; Bendo et al. 2006; Galametz et al. 2009, 2011; Israel et al. 2010; Bot et al. 2010; Dale et al. 2012; Planck Collaboration 2011a). Several reasons have been given to explain this excess: the existence of a large amount of cold $(<10 \mathrm{~K})$ dust (Galliano et al. 2003, 2005; Galametz et al. 2009, 2011), different dust grain properties (Lisenfeld et al. 2002; Reach et al. 1995; Meny et al. 2007), or magnetic nanograins, which can produce magnetic dipole radiation at microwave and submm wavelengths (Draine \& Hensley 2012). 
It is also noteworthy that one sees a variety of FIR colours for gas-rich dwarf galaxies, with examples of warm FIR SEDs (as found from IRAS colours - e.g. Melisse \& Israel 1994) or of cold FIR SEDs (as revealed by ISO and Herschel measurements extending longwards of $100 \mu \mathrm{m}-$ e.g. Popescu et al. 2002; Grossi et al. 2010). Such variety may not be too surprising given that the SF in dwarf galaxies is likely to be fundamentally bursty in nature. If we interpret the FIR SED in terms of the combination of cooler distributed cirrus dust emission and warm dust emission from grains in SF regions, the latter opaque structures should be most prominent in the early stages of the evolution of a starburst leading to a warm FIR SED. Over time the starburst fades, leaving the cirrus component and a cooler FIR SED. Other factors potentially contributing to the observed variety in FIR colours are differences in the contribution of very small grains (e.g. Galliano et al. 2003, 2005) and the possible presence of extended cold dust outside the main star forming disk of the galaxy (Popescu et al. 2002).

To interpret the dust SED of a galaxy and to understand the differences in the SEDs of dwarf galaxies, a physical model based on realistic dust properties and considering the heating and emission of dust immersed in a wide range of interstellar radiation fields (ISRFs) is needed. Ideally, radiation transport in a realistic geometry should be done, but this is often difficult owing to the complex geometry and large number of parameters. Models can generally be classified into three broad groups: (1) modified blackbody fits, which are too simple to describe reality correctly but give a first idea of the dust temperature ranges; (2) semi-empirical models that try, in a simplified way, to describe dust immersed in a range of different ISRFs (e.g. Dale et al. 2001; Draine et al. 2007; Galametz et al. 2009, 2011; da Cunha et al. 2008; Natale et al. 2010); and (3) models that include full radiation transfer (e.g. Popescu et al. 2011 for spiral galaxies; Siebenmorgen \& Krügel 2007 for starburst galaxies; see also Silva et al. 1998; Popescu et al. 2000, 2004; Misiriotis et al. 2001; Bianchi 2008; Baes et al. 2010, 2011 and MacLachlan et al. 2011), which are the most precise description of a galaxy if all parameters, including the geometry, are known.

The new IR facilities with their high angular resolution and sensitivity make, for the first time, detailed comparisons between models and data possible. For nearby, hence spatially resolved, galaxies, the spatial variations of the dust SED can be probed and modelled. In particular, a good spatial resolution permits to separate and treat the emission from dust heated by the UV radiation of massive stars in HII regions, their adjacent photodissociation regions (PDR), and the diffuse dust heated by the general ISRF.

We chose NGC 4214 as a test case because of the large amount of ancillary data and its proximity $\left(1^{\prime \prime} \sim 14 \mathrm{pc}, D=\right.$ 2.9 Mpc; Maíz-Apellániz et al. 2002), which allows us to observationally separate the dust emission from the two prominent central SF complexes and from the diffuse dust component in the disk. This allows us to apply a radiation transfer treatment constrained by measurements of direct light in UV to NIR and dust/PAH re-radiated light in both the diffuse and SF complexes.

We chose the radiation transfer model of Popescu et al. (2011) for spiral galaxies since this self-consistently treats the dust emission from the diffuse and SF complex components, considering the illumination of diffuse dust both by the smoothly distributed stellar populations and by escaping light from the HII regions in spiral disks. Although NGC 4214 is a dwarf galaxy, rather than a spiral galaxy, it nevertheless exhibits the main geometrical features of the Popescu et al. (2011) model. In particular, NGC 4214 shows an exponential disk distribution of the stellar light, the diffuse dust emission and the atomic gas emission, so it is actually a good candidate to be fitted with a model for disk galaxies. While maintaining consistency within the framework of the Popescu et al. (2011) model, we use the model of Groves et al. (2008) to provide a detailed description of the dust emission from the HII regions and their surrounding PDRs on scales of tens of pc. These PDRs mark the transition from the ionized medium to the dense molecular gas left over from the highly opaque cloud out of which the stars formed. The model of Groves et al. (2008) considers the dynamical evolution of the distance of the PDRs from the central ionizing star clusters due to the mechanical effect of stellar winds on the surrounding ISM. This model is therefore particularly suitable to describing the two prominent SF complexes in the centre of NGC 4214, since these show shell structures imaged by Hubble Space Telescope (HST) surrounding the two main star clusters of the galaxy, which may be delineating wind blown bubbles around the HII regions.

NGC 4214 is a Magellanic starbursting dwarf irregular galaxy (de Vaucouleurs et al. 1991a) which shows a large degree of structure, from the HI holes and shells (McIntyre 1998) typical of dwarf galaxies (Walter \& Brinks 1999) to clear indications of a spiral pattern and a central bar. The molecular gas traced by the $\mathrm{CO}(1-0)$ line shows three well-differentiated $\mathrm{CO}$ emitting complexes related to the main SF complexes with a total molecular gas mass of $M_{\mathrm{H} 2}=5.1 \times 10^{6} M_{\odot}$ (Walter et al. 2001, obtained with a Galactic conversion factor). NGC 4214 is a gasrich galaxy, the total mass of atomic gas is $M_{\mathrm{HI}}=4.1 \times 10^{8} M_{\odot}$ (Walter et al. 2008).

Karachentsev et al. (2004) report a stellar mass of $\sim 1.5 \times$ $10^{9} M_{\odot}$ for NGC 4214, similar to the mass found in the Large Magellanic Cloud (LMC). Ultraviolet (UV), optical, and nearinfrared (NIR) images of NGC 4214 show that the young stellar population is embedded in a smooth disk of old stars (see the large field of Fig. 1), which can account for a significant fraction $(\sim 75 \%)$ of the total stellar mass (Williams et al. 2011). Despite this high fraction of old stars, NGC 4214 is a galaxy with an intense, recent SF activity, as shown by the two star forming complexes located in its centre and frequently referred to in the literature as NGC 4214-NW and NGC 4214-SE. When resolved, the two complexes show smaller individual knots of SF (see the small field of Fig.1). One of the most striking features in these complexes is the large shell structure in the NW region, where most of the gas in front of the central star cluster seems to have been removed by the action of stellar winds and supernovae (SNe) (Maíz-Apellániz et al. 1998; MacKenty et al. 2000). NGC 4214-SE is more compact and shows no clear evidence of a decoupling between the star clusters and the gas. The morphological differences for the two complexes were interpreted as an evolutionary trend by MacKenty et al. (2000). Using stellar synthesis models, Úbeda et al. (2007) determined the age, the mass, the radius, and the extinction of the star clusters within the NW and SE complexes. They found an age of $5 \mathrm{Myr}$ for the star clusters in the NW region, whereas the age of the star clusters of the $\mathrm{SE}$ region ranges from 1.7 to $4.0 \mathrm{Myr}$.

The internal extinction distribution of the two complexes was studied by Maíz-Apellániz et al. (1998). These authors found that the distribution of the dust clouds is correlated to the distribution of the ionized gas: the dust is located at the border of the star clusters in the NW complex, whereas for the SE region the dust clouds seem to be co-spatial with the star clusters. The metallicities of the two complexes have been measured by Kobulnicky \& Skillman (1996), who found values in the range of $Z \sim 0.3 Z_{\odot}$ with little dispersion. 


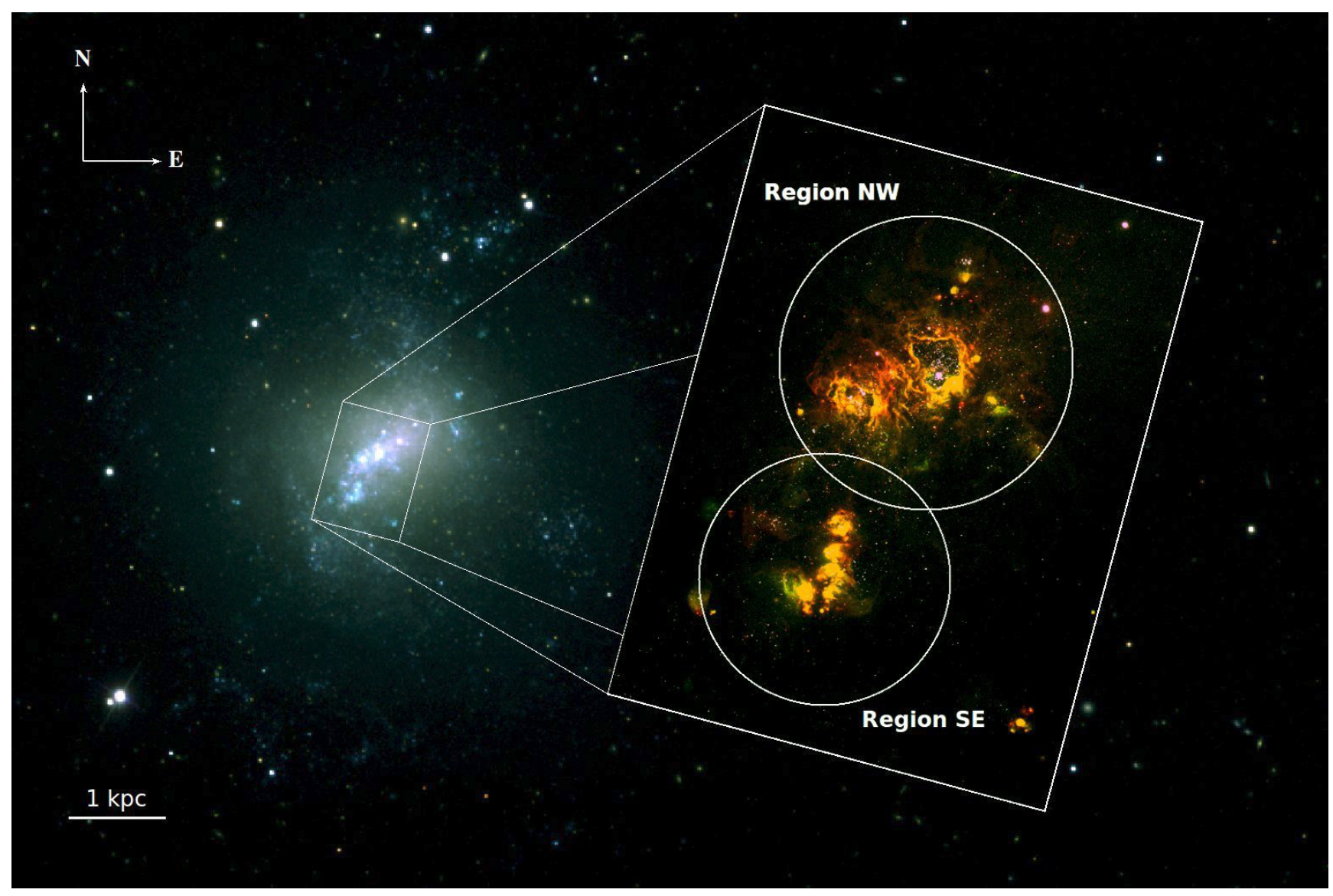

Fig. 1. Combined SLOAN and HST image of NGC 4214. Large field: image of NGC 4214 from a RGB combination of SLOAN r, g, and u bands. Zoom-in: image of the centre of NGC 4214 from a RGB combination of HST-WFC3 filters F657N ( $\mathrm{H}_{\alpha}+$ cont), F502N ([OIII] + cont) and F336W (cont). The two circles are the apertures that we use for the aperture photometry of the regions NW and SE.

Thus, the large number and the broad range of previous studies performed for this galaxy, as well as the significant amount of multiwavelength data, give us the opportunity to obtain an accurate picture of the stellar, dust, and gas components in NGC 4214.

\section{The data}

A wide range of data exists in archives for NGC 4214, from the UV to the submm. The entire dust emission SED from the MIR to the submm is covered by data from Spitzer, Herschel, and Planck. In addition, to extend the dust SED to the mm range we have mapped the galaxy at $1.2 \mathrm{~mm}$ with the IRAM $30 \mathrm{~m}$ telescope. In the following sections we describe the data and the reduction that we performed prior to extracting the photometry. A selection of images at different wavelengths is shown in Fig. 2.

\subsection{GALEX}

NGC 4214 was observed with GALEX on January 13, 2010, as part of the programme GI4-095 (Janice Lee, 2009). Farultraviolet (FUV) and near-ultraviolet (NUV) photometric maps were obtained.

The GALEX FUV detector has an effective wavelength of $1539 \AA$, a bandwidth of $442 \AA$, and an image resolution of $4.2^{\prime \prime}$. For the NUV detector, the effective wavelength is $2316 \AA$, the bandwidth $1060 \AA$, and the image resolution 5.3". With both detectors the two main SF complexes NW and SE are resolved well and the field of view, with a radius of about $36^{\prime}$, covers the entire disk of NGC 4214. We have retrieved the calibrated maps from the archive and subtracted the background using the corresponding maps from the archive.

\subsection{HST-WFC3}

Wide Field Camera 3 (WFC3) data were obtained for NGC 4214 as part of the WFC Science Oversight Committee (SOC) Early Release Science (ERS) programme (programme ID11360, P.I. Robert O'Connell). A total of seven stellar and seven nebular images were taken using UVIS and IR cameras. Both cameras onboard the HST have a field of view of $\sim 2^{\prime}$, and they cover the central star forming complexes NW and SE, allowing us to separate them into several smaller star forming regions. We obtained calibrated maps for the stellar filters F225W, F336W, F438W, F547M, F814W, F110W, and F160W from the HST Data Archive.

\subsection{Spitzer}

The Spitzer data for NGC 4214 used in this work are part of the Local Volume Legacy (LVL) survey (Dale et al. 2009). The LVL sample contains 258 galaxies within $11 \mathrm{Mpc}$, which have been mapped with both MIPS (3 bands) and IRAC (4 bands).

For MIPS data the exposure times were 146.8, 83.8, and $16.76 \mathrm{~s}$ at 24,70 , and $160 \mu \mathrm{m}$, respectively. The area mapped in the three bands, approximately $16^{\prime} \times 16^{\prime}$, covers the whole disk of NGC 4214. The resolution of $6^{\prime \prime}$ for MIPS $24 \mu \mathrm{m}$ allows us 

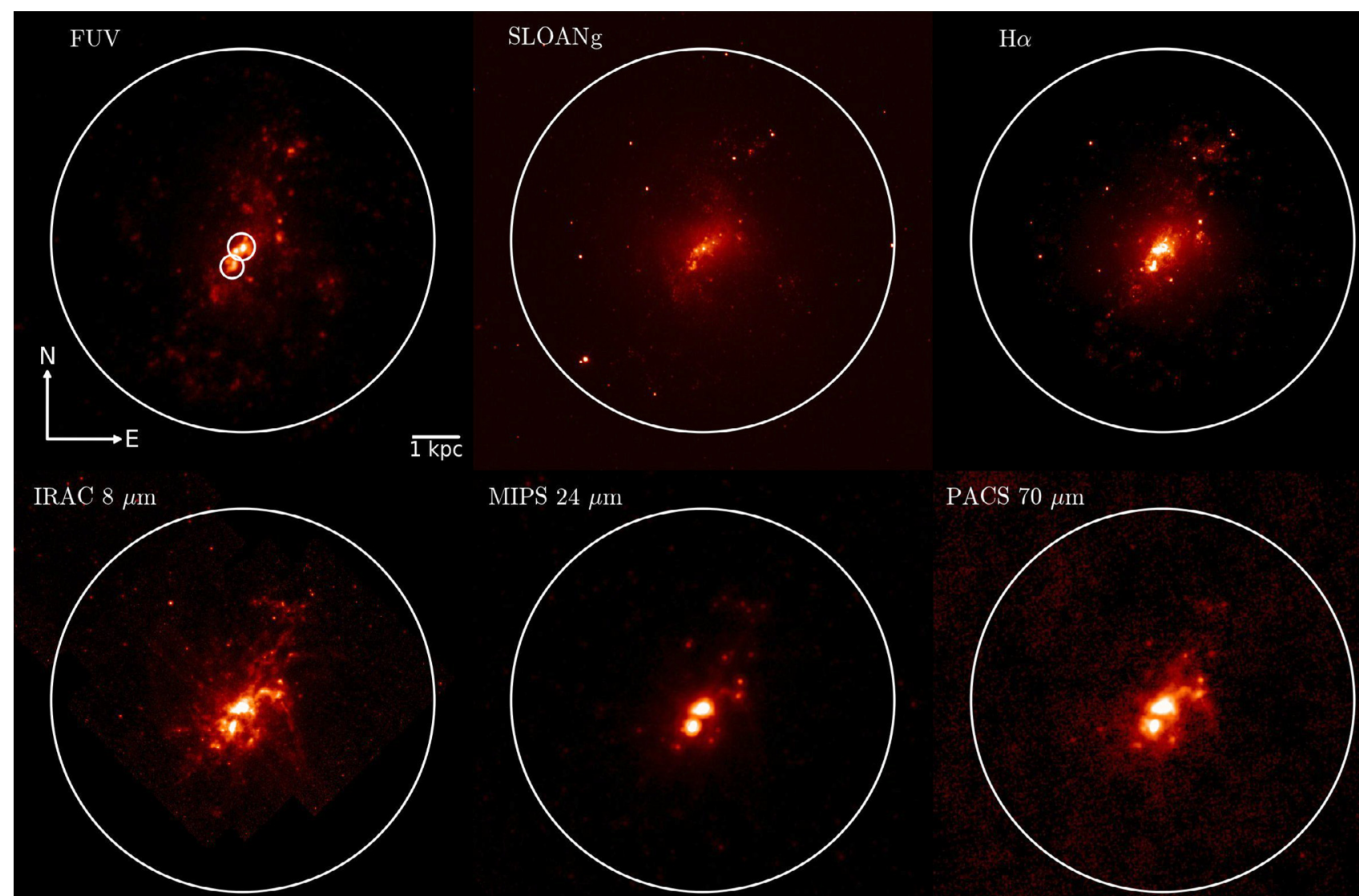

\section{MIPS $24 \mu \mathrm{m}$}

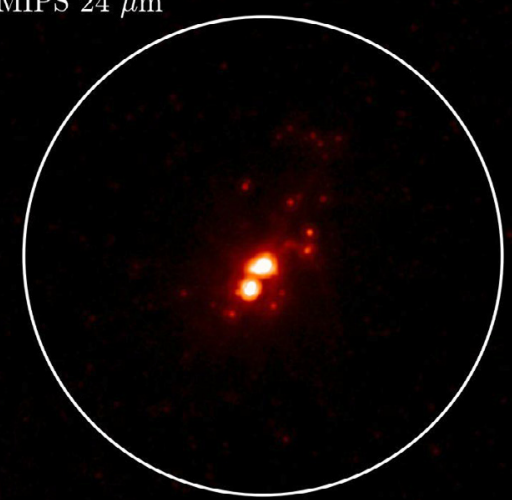

\section{PACS $70 \mu \mathrm{m}$}

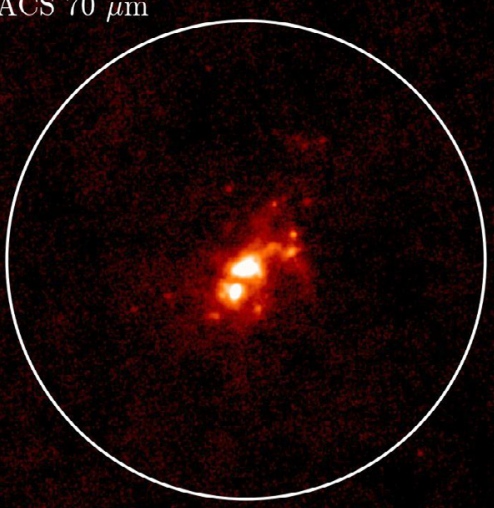

PACS $100 \mu \mathrm{m}$

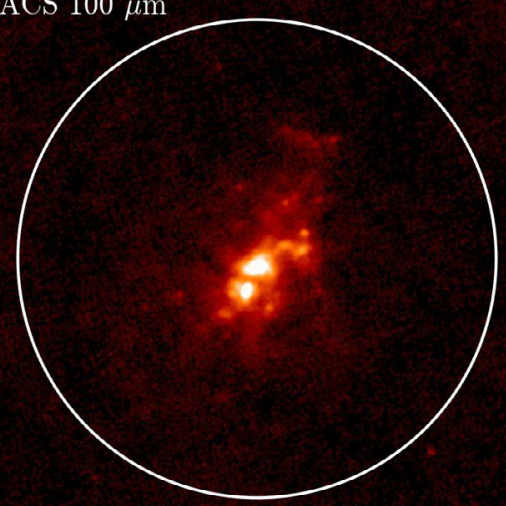

PACS $160 \mu \mathrm{m}$

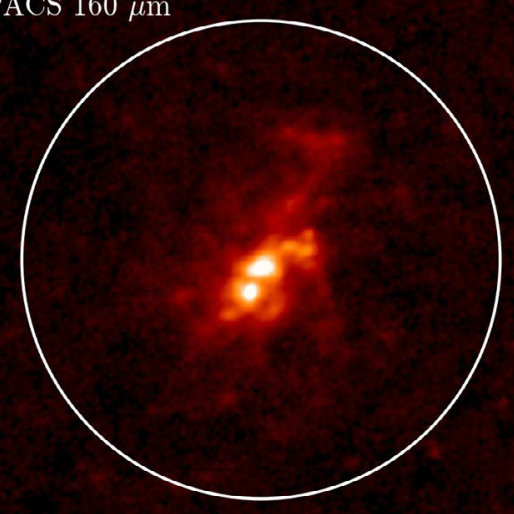

SPIRE $250 \mu \mathrm{m}$

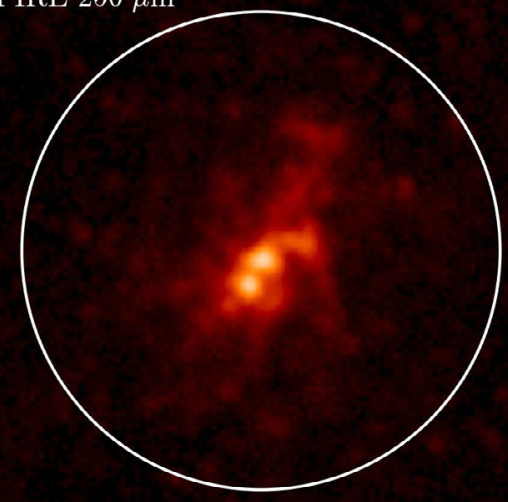

\section{SPIRE $350 \mu \mathrm{m}$}

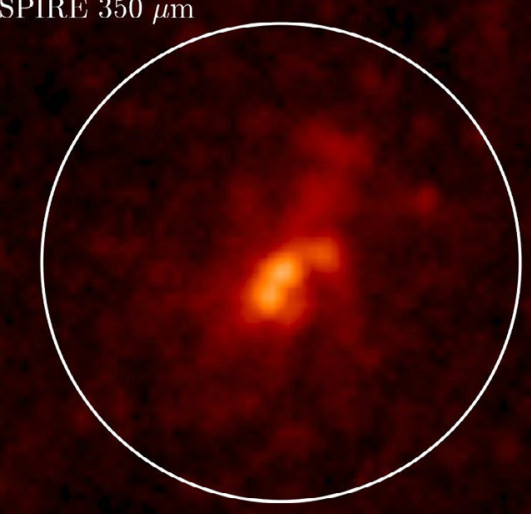

SPIRE $500 \mu \mathrm{m}$

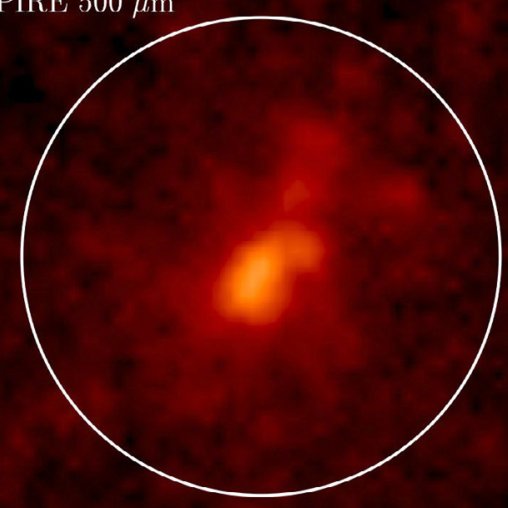

\section{MAMBO}

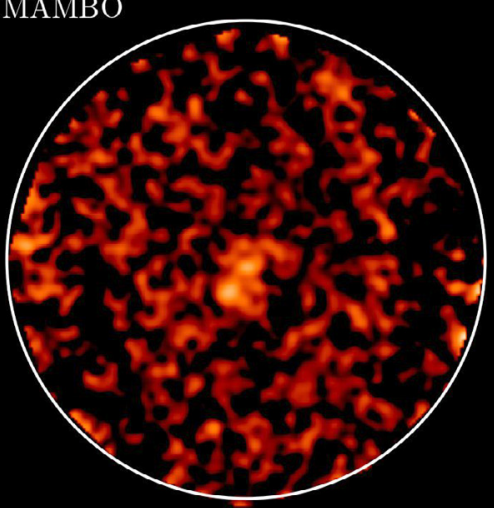

Fig. 2. Images of NGC 4214 at different wavelengths. The large circle shows the aperture that we chose for the entire disk emission and the smaller circles in the top-left panel the apertures to measure the emission from the HII regions NW and SE. 
to determine fluxes individually for the SF complexes NW and SE. This separation is not possible for MIPS 70 and $160 \mu \mathrm{m}$ due to a poorer (18" and 38", respectively) angular resolution. We obtained the images from the archive and subtracted the background, which we determined as the mean value of an annulus located outside the disk of the galaxy.

As for several other galaxies in the LVL sample, IRAC data of NGC 4214 was taken from previous Spitzer programmes. In the case of NGC 4214, the LVL makes use of the IRAC data from the Mid-IR Hubble Atlas of Galaxies (Fazio \& Pahre 2004). As part of this programme, NGC 4214 was observed with IRAC at 3.6, 4.5, 5.8, and $8.0 \mu \mathrm{m}$ in May 2004. In our study of the star forming regions, we only used the IRAC $3.6 \mu \mathrm{m}$ band, which we assumed as pure stellar emission, and the IRAC $8.0 \mu \mathrm{m}$ band. To isolate the dust component from the IRAC $8.0 \mu \mathrm{m}$, we subtracted the stellar emission using the IRAC $3.6 \mu \mathrm{m}$ image and applying the formula provided in Helou et al. (2004). With an angular resolution of 1.7" and 2.0" for the IRAC 3.6 and $8.0 \mu \mathrm{m}$ bands, respectively, the SF complexes NW and SE are resolved into several smaller HII regions.

The total mapped area by IRAC, approximately of $13^{\prime} \times 6^{\prime}$, covered the central part of the disk of NGC 4214, but not the entire extended disk. Therefore, the total emission at 3.6 and $8 \mu \mathrm{m}$ that we obtain might be underestimated. However, judging from the IRAC $8.0 \mu \mathrm{m}$ image in Fig. 2, we believe that the underestimate is not significant.

The uncertainty in the flux calibration is better than $10 \%$ for all IRAC (Reach et al. 2005; Fazio et al. 2004) and MIPS $24 \mu \mathrm{m}$ bands (Engelbracht et al. 2007), and is better than 20\% for MIPS 70 and $160 \mu \mathrm{m}$ bands (Rieke et al. 2004). We adopt these numbers as the calibration uncertainties in our error estimates for the fluxes (see Sect. 3.5).

\subsection{Herschel}

NGC 4214 was observed by Herschel's PACS and SPIRE instruments as part of the Dwarf Galaxy Survey programme (PI. S. Madden), a guaranteed time key programme with the aim of mapping the dust and gas in 51 nearby dwarf galaxies.

SPIRE data were obtained on June 28, 2010, and cover a circular area of approximately $17^{\prime}$. We retrieved the HIPE Level 2.0 images from the archive and performed further analysis for our purposes. Thanks to their good quality and the absence of artefacts, we only subtracted the background, which we measured in the same annulus used for MIPS data. The resolution of $18^{\prime \prime}$ at SPIRE $250 \mu \mathrm{m}$ allowed us to measure individual fluxes for the two star forming complexes. This distinction was not possible in the case of SPIRE 350, and $500 \mu \mathrm{m}$ due to a poorer image resolution (25" and 37", respectively). Swinyard et al. (2010) reported for SPIRE 250, 350, and $500 \mu \mathrm{m}$ an uncertainty in the flux calibration of $15 \%$, which we adopted in our error calculation.

PACS data were obtained on December 27 and 28, 2010 and cover an area of $\sim 25^{\prime} \times 25^{\prime}$. The data from the archive were processed to level 1 with HIPE 8.2.0. To complete the reduction we used the Scanamorphos (Roussel 2012) map-making technique. The background was subtracted from the final maps using the same annulus as mentioned before. At the resolution of PACS at 70,100 , and $160 \mu \mathrm{m}\left(5.2^{\prime \prime}, 7.7^{\prime \prime}\right.$, and $12^{\prime \prime}$, respectively), the two star forming complexes are resolved. The uncertainty in the flux calibration is of the order of $10 \%$.

\subsection{Planck}

The Planck Early Release Compact Source Catalogue includes three detections of NGC 4214 at 350, 550, and $850 \mu \mathrm{m}$, all of them composed of two different observations. For the three detections, the subtracted cosmic microwave background (CMB) flux was less than $30 \%$ of the total flux measured originally by Planck. The catalogue gives two estimates for the total flux of a source: i) FLUX, obtained by aperture photometry within the nominal sky-averaged FWHM, which is $4.45^{\prime}, 4.71^{\prime}$, and $4.62^{\prime}$ for 350,550 , and $850 \mu \mathrm{m}$, respectively; and ii) FLUXDET, obtained by their native detection algorithm (Planck Collaboration 2011b). We adopt here the first flux estimate, FLUX, which is recommended for sources that are point-like with respect to the Planck beam, as is the case for NGC 4214. We use the difference between the two estimates (which is larger than the nominal error of each flux) as an estimate of the errors.

\subsection{IRAM $30 \mathrm{~m}$}

Several observations at $1200 \mu \mathrm{m}$ were made by our group between December 2009 and November 2010 at the IRAM 30 m telescope on Pico Veleta (Spain), with the 37-channel bolometer array of the Max-Planck-Institut für Radioastronomie (MPIfR). The 37 pixels are located in a hexagonal structure with a beamsize of $10.8^{\prime \prime}$ and a pixel-to-pixel separation of about $23^{\prime \prime}$. The observations were done on-the-fly, and they were calibrated by observations of the planet Mars and secondary calibrators. Observations were reduced and combined into an image with Robert Zylka's MOPSIC ${ }^{1}$ pipeline in a standard manner, including baseline subtraction, and spike and sky noise removal. We smoothed the combined image to a resolution of $18^{\prime \prime}$ in order to increase the signal-to-noise ratio. At this resolution, the two main star forming complexes are still resolved. Our observations were not sensitive enough to detect any extended emission outside the two main SF complexes. We adopted $30 \%$ calibration uncertainty.

\subsection{Thermal radio emission}

The thermal radio emission in an HII region is proportional to the production rate of Lyman continuum photons $N_{\text {Lyc }}$. Condon (1992) gives the following relation between the thermal radio emission and the extinction-corrected $\mathrm{H} \beta$ line flux, $F(\mathrm{H} \beta)$, which is proportional to $N_{\text {Lyc }}$ :

$$
\frac{S_{\mathrm{T}}}{\mathrm{mJy}} \sim \frac{1}{0.28}\left(\frac{T}{10^{4} \mathrm{~K}}\right)^{0.52}\left(\frac{v}{\mathrm{GHz}}\right)^{-0.1} \frac{F(\mathrm{H} \beta)}{10^{-12} \mathrm{erg} \mathrm{cm}^{-2} \mathrm{~s}^{-1}},
$$

where $S_{T}$ is the thermal flux density at the frequency $v$ for an HII region with a temperature $T$.

We used the measurements of the $\mathrm{H} \alpha$ emission from MacKenty et al. (2000) to calculate the expected thermal radio emission from NW and SE. They presented an $\mathrm{H} \alpha$ narrowband map of the centre of NGC 4214 obtained with the HST Wide Field and Planetary Camera (WFPC2). They used the spectroscopic values of the Balmer ratio $\mathrm{H} \alpha / \mathrm{H} \beta$ presented by Maíz-Apellániz (2000) to correct for dust extinction and they reported intrinsic $\mathrm{H} \alpha$ fluxes of NW and SE. Assuming that the dust is mixed well with the gas, MacKenty et al. (2000) calculated extinction-corrected $\mathrm{H} \alpha$ fluxes of $12.88 \times 10^{-12} \mathrm{erg} \mathrm{cm}^{-2} \mathrm{~s}^{-1}$ for the NW complex and $5.75 \times 10^{-12} \mathrm{erg} \mathrm{cm}^{-2} \mathrm{~s}^{-1}$ for the SE complex.

\footnotetext{
1 See http://www.iram.es/IRAMES/mainWiki/CookbookMopsic
} 
For the NW region, MacKenty et al. (2000) used a circular aperture of radius $20.6^{\prime \prime}$, practically identical to the aperture used in this work (see Sect. 3.1). For the SE region they used a smaller aperture $\left(9.96^{\prime \prime}\right)$. We therefore multiplied their flux by a factor 1.4 , corresponding to the ratio of the uncorrected $\mathrm{H} \alpha$ fluxes measured from the same image in apertures of 9.96" and $18^{\prime \prime}$, respectively. With these extinction-corrected $\mathrm{H} \alpha$ fluxes, the intrinsic $\mathrm{H} \alpha / \mathrm{H} \beta$ ratio, and the temperature of $10^{4} \mathrm{~K}$ reported by Kobulnicky \& Skillman (1996), we obtain with Eq. (1) flux densities at $8.46 \mathrm{GHz}(3.5 \mathrm{~cm})$ of $13 \mathrm{mJy}$ for $\mathrm{NW}$ and $8 \mathrm{mJy}$ for SE.

The combined prediction for the thermal radio emission of NW and SE (21 mJy) can be roughly compared with the measurement from the VLA at $8.46 \mathrm{GHz}$ of $24.2 \pm 4.8 \mathrm{mJy}$ given by Kepley et al. (2011) for the total radio emission, which includes synchrotron and thermal emission. From the uncorrected $\mathrm{H} \alpha$ emission, Kepley et al. (2011) estimate that the fraction of thermal emission is on average $\sim 0.5$ (see Fig. 9 in their paper). However, since these authors did not correct the $\mathrm{H} \alpha$ emission for dust attenuation, their estimation gives a lower limit of the thermal fraction. Our estimation of free-free emission (21 mJy) falls within the limits of thermal fractions of 0.5 (12.1 mJy) and $1.0(24.2 \mathrm{mJy})$ and is thus consistent with their data. We used their limits to roughly estimate an error of the thermal radio flux of $\sim 30 \%$.

\section{Photometry}

We need to separate the dust emission from the two major SF complexes NW and SE and the diffuse disk, in order to establish the individual SEDs of these morphological components. We therefore independently carried out aperture photometry for the regions SE and NW and for the total emission of the entire galaxy. We determined the diffuse dust emission as the difference between the total emission and the sum of the emissions of SE and NW regions.

Prior to extracting the photometry, we regridded all the images to a common pixel size keeping the original resolution of each image. Then, corresponding aperture corrections were applied to the fluxes in each band. In this section we describe how we performed the aperture photometry, which corrections were applied, and how the errors were handled.

\subsection{Apertures}

We chose the apertures around the HII regions to be large enough to enclose the entire localized dust emission and simultaneously tried to include as little emission from the diffuse component as possible. Mainly based on the MIPS $24 \mu \mathrm{m}$ image, we chose apertures with radii of $21^{\prime \prime}$ for the NW and $18^{\prime \prime}$ for the SE complex (see Figs. 1 and 2). The $24 \mu \mathrm{m}$ image was used since it is the filter that is expected to most faithfully trace the warm dust in thermal equilibrium with the strong radiation fields from the star clusters in the PDRs and HII regions associated with the SF regions. We were able to measure the emission from each SF complex only for those wavelengths where both complexes were spatially resolved, which excluded the maps of MIPS 70 and $160 \mu \mathrm{m}$ and SPIRE 350 and $500 \mu \mathrm{m}$. The measured, background-subtracted fluxes are listed in Col. 3 in Tables 1 for NW $\left(F_{\mathrm{NW}}^{\text {sub }}\right)$ and 2 for $\mathrm{SE}\left(F_{\mathrm{SE}}^{\mathrm{sub}}\right)$.

One issue of particular relevance to the quantitative analysis presented in this paper is that of the subtraction of the local background underlying the NW and SE SF regions. Ideally, we need to cleanly separate the localized dust/PAH emission associated with the HII regions and the local PDRs on scales of tens of parsecs (marking the interface between the tenuous gas ionized by the star cluster and the dense material of the parent molecular clouds) from the underlying extended emission from diffuse dust distributed on the kpc scale of the disk. In practice it is very challenging to perform such a separation, not only because of blending effects due to the finite angular resolution (these are quantified through the aperture corrections described in Sect. 3.2, below), but also because of the limited range of UV photons that have escaped the SF regions and have passed into the diffuse dust layer, forming a halo around the SF regions.

The extent and brightness of this diffuse halo emission is expected to vary strongly with infrared wavelength in a way that is both difficult to measure directly and also uncertain to predict theoretically on an a priori basis. Therefore, for simplicity, we have opted in this paper for making no attempt to subtract the underlying diffuse background when performing aperture photometry on the SF regions. For future reference we simply note here that we may thereby be overestimating the flux densities of the NW and SE SF regions, most particularly in the PAH bands, but also to some extent around the peak of the SED in the FIR. The effect at $24 \mu \mathrm{m}$, however, is likely to be small, since, as already noted, this band is primarily sensitive to warm dust heated by the intense radiation fields in the PDRs and HII regions close to the star clusters.

For the measurement of the total emission of NGC 4214 we chose an aperture of $5^{\prime}$ in radius (see Fig. 2). We tested with growth curves at all wavelengths that this aperture enclosed the entire emission of the galaxy. For the Planck measurements, which include the total emission of the galaxy, the apertures could not be chosen. However, their values are very close (see Sect. 2.5) to ours, and our growth curves show that at the radius of the Planck beam the emission was enclosed completely.

The total emission from the MAMBO $1.2 \mathrm{~mm}$ map is more uncertain owing to the poor signal-to-noise ratio at the outer parts of the area covered by our observations, which is practically the same as the aperture of $5^{\prime}$ used for the other images. In the moderately smoothed image (resolution 18", shown in Fig. 2), no diffuse emission can be seen. However, when we smooth the map to an angular resolution of $40^{\prime \prime}$, diffuse dust emission becomes visible in the inner 160" whose structure corresponds very well to the SPIRE $500 \mu \mathrm{m}$ map. Beyond this radius we are not sure that the structures are real. The growth curve of MAMBO keeps rising until 200" where it becomes approximately flat. We obtained integrated fluxes of $0.26 \pm 0.03 \mathrm{Jy}$ for the aperture of $160^{\prime \prime}$ and $0.35 \pm 0.11 \mathrm{Jy}$ for $300^{\prime \prime}$. The lower value should represent a reliable lower limit for the total flux in NGC 4214. The total flux is more uncertain, however, given the flatness of the growth-curve beyond 200", we are confident that the flux at $300^{\prime \prime}$ is a reasonable estimate. Due to these uncertainties, we did not include the MAMBO data point in our fitting procedure, but we show it in the figures for comparison with the models.

The measured, background-subtracted fluxes of the total emission are listed in Col. 3 of Table 3.

\subsection{Aperture correction}

Because of the different resolutions of the images we applied aperture corrections to the NW and SE IR fluxes. We derived values of the aperture correction by adopting the IRAC $8 \mu \mathrm{m}$ as the high-resolution model of the flux distribution. We first measured the flux within the NW and SE apertures in the IRAC $8 \mu \mathrm{m}$ 
I. Hermelo et al.: The dust SED of dwarf galaxies. I.

Table 1. Flux densities for region NW obtained for an aperture of $21^{\prime \prime}$ radius centred at RA $=12 \mathrm{~h} 15 \mathrm{~m} 39.6 \mathrm{~s}$ Dec $=+36^{\circ} 19^{\prime} 36^{\prime \prime} .5(\mathrm{~J} 2000)(\mathrm{see}$ Figs. 1 and 2).

\begin{tabular}{lccccc}
\hline \hline Band & $\lambda_{0}(\mu \mathrm{m})$ & $F_{\mathrm{NW}}^{\text {sub }}(\mathrm{Jy})$ & $C_{\text {apert }}$ & $C_{\text {color }}$ & $F_{\mathrm{NW}}(\mathrm{Jy})$ \\
\hline IRAC8 & 7.872 & 0.163 & 1.000 & 0.590 & $0.276 \pm 0.035$ \\
MIPS24 & 23.680 & 0.733 & 1.078 & 0.986 & $0.801 \pm 0.103$ \\
PACS70 & 70.000 & 7.612 & 1.039 & 0.982 & $8.053 \pm 1.031$ \\
PACS100 & 100.000 & 8.443 & 1.050 & 0.985 & $9.000 \pm 1.153$ \\
PACS160 & 160.000 & 6.114 & 1.075 & 1.010 & $6.507 \pm 0.833$ \\
SPIRE250 & 250.000 & 2.329 & 1.110 & 0.992 & $2.605 \pm 0.443$ \\
MAMBO $^{*}$ & 1200.000 & 0.031 & 1.067 & 1.000 & $0.033 \pm 0.010$ \\
$S_{\mathrm{T}}(0.5 \mathrm{~cm})$ & 5000.000 & 0.011 & 1.000 & 1.000 & $0.011 \pm 0.003$ \\
\hline
\end{tabular}

Notes. $F_{\mathrm{NW}}^{\mathrm{sub}}$ is the measured, background-subtracted flux and $F_{\mathrm{NW}}$ is the final flux, after applying aperture and colour corrections. ${ }^{(*)}$ The contribution from thermal radio emission was not subtracted.

Table 2. Flux densities for region SE obtained for an aperture of $18^{\prime \prime}$ radius centred at RA $=12 \mathrm{~h} 15 \mathrm{~m} 40.8 \mathrm{~s}$ Dec $=+36^{\circ} 19^{\prime} 05^{\prime \prime} .5(\mathrm{~J} 2000)(\mathrm{see}$ Figs. 1 and 2).

\begin{tabular}{lccccc}
\hline \hline Band & $\lambda_{0}(\mu \mathrm{m})$ & $F_{\mathrm{SE}}^{\text {sub }}(\mathrm{Jy})$ & $C_{\text {apert }}$ & $C_{\text {color }}$ & $F_{\mathrm{SE}}(\mathrm{Jy})$ \\
\hline IRAC8 & 7.872 & 0.105 & 1.000 & 0.590 & $0.178 \pm 0.023$ \\
MIPS24 & 23.680 & 0.602 & 1.069 & 0.986 & $0.653 \pm 0.084$ \\
PACS70 & 70.000 & 4.659 & 1.032 & 0.982 & $4.896 \pm 0.627$ \\
PACS100 & 100.000 & 5.416 & 1.040 & 0.985 & $5.719 \pm 0.732$ \\
PACS160 & 160.000 & 4.094 & 1.057 & 1.010 & $4.285 \pm 0.549$ \\
SPIRE250 & 250.000 & 1.689 & 1.078 & 0.992 & $1.835 \pm 0.312$ \\
MAMBO $^{*}$ & 1200.000 & 0.029 & 1.021 & 1.000 & $0.029 \pm 0.009$ \\
$S_{\mathrm{T}}(0.5 \mathrm{~cm})$ & 5000.000 & 0.005 & 1.400 & 1.000 & $0.007 \pm 0.002$ \\
\hline
\end{tabular}

Notes. $F_{\mathrm{SE}}^{\text {sub }}$ is the measured, background-subtracted flux and $F_{\mathrm{SE}}$ is the final flux, after applying aperture and colour corrections. ${ }^{(*)}$ The contribution from thermal radio emission was not subtracted.

image. Then we convolved the IRAC $8 \mu \mathrm{m}$ image with the point-spread-function (PSF) of MIPS 24, PACS 70, PACS 100, PACS 160, SPIRE 250, and MAMBO, and measured the flux again within the same apertures. The ratio between the flux measured in the original and convolved images gives the aperture correction for each band. The values are listed in Col. 4 of Tables 1 and 2 .

\subsection{Colour corrections}

To directly compare the monochromatic fluxes with the models of Popescu et al. (2011) and Groves et al. (2008), we applied colour corrections to the IRAC, MIPS, PACS, SPIRE, and Planck fluxes. We followed the procedures described in the corresponding manuals ${ }^{2}$, where colour correction factors for different source distributions are listed.

We chose the colour correction factors for the spectrum that most closely resembled the model spectrum at a given wavelength. For the MIPS $24 \mu \mathrm{m}$ band we adopted a blackbody with a temperature of $70 \mathrm{~K}$; for the MIPS $70 \mu \mathrm{m}$, MIPS $160 \mu \mathrm{m}$ and PACS bands a blackbody with a temperature of $50 \mathrm{~K}$; for SPIRE bands and Planck $350 \mu \mathrm{m}$ we chose a power-law spectrum with $\alpha=+2.0$; for Planck $550 \mu \mathrm{m}$ a power-law spectrum with $\alpha=+2.5$; and for Planck $850 \mu \mathrm{m}$ a power-law spectrum with $\alpha=+3.0$ (where the exponent $\alpha$ is defined as $F_{v} \propto v^{\alpha}$ ). For the particular case of IRAC $8 \mu \mathrm{m}$, we adopted the colour correction for a PAH-dominated spectrum. At $8 \mu \mathrm{m}$ we additionally compared model and data by generating synthetic integrated

2 See the IRAC Instrument Handbook, MIPS Instrument Handbook,
PACS Observer's Manual, SPIRE Observers' Manual and Planck
Collaboration (2011) for IRAC, MIPS, SPIRE and Planck, respectively. fluxes. For this we (i) multiplied the model spectrum with the $8 \mu \mathrm{m}$ filter profile and integrated it over the $8 \mu \mathrm{m}$ filter band and (ii) multiplied the observed $8 \mu \mathrm{m}$ data, adopting the blackbody of $T=10^{4} \mathrm{~K}$ profile on which the flux definition of IRAC is based, with the $8 \mu \mathrm{m}$ profile and integrated it over the $8 \mu \mathrm{m}$ filter band. The comparison of both values allowed an alternative estimate of the goodness of the fit, based entirely on the data and the model spectrum. We found that this comparison produced within $10 \%$ the same ratios as the comparison between colour-corrected data point and model flux.

The colour corrections are listed in Tables 1-3.

\subsection{Line contamination}

Most of the FIR/submm broad-band filters used for this work are contaminated by molecular or atomic emission lines. In the cases where data were available, we estimated the line contamination by comparing the luminosity of the emission line with the total luminosity measured in the filter, obtained by integrating the product of the source spectrum and the spectral response of the filter over the frequency range of the filter band.

Emission lines from different $\mathrm{CO}$ rotational transitions fall into SPIRE, Planck, and MAMBO filters. We used the CO(1-0) map presented by Walter et al. (2001) as a reference. We determined a velocity integrated flux of $9.3 \mathrm{Jy} \mathrm{km} \mathrm{s}^{-1}$ in the NW region and $12.8 \mathrm{Jy} \mathrm{km} \mathrm{s}^{-1}$ in the SE region. The MAMBO filter band, with a bandwidth of about $80 \mathrm{GHz}$, is affected by the $\mathrm{CO}(2-1)$ line. We estimated the flux of the $\mathrm{CO}(2-1)$ line from the measured $\mathrm{CO}(1-0)$ value assuming that the line intensity ratio it is given by the value for optically thick, thermalized $\mathrm{CO}$, $I_{\mathrm{CO}(1-0)} / I_{\mathrm{CO}(2-1)}=1$. The contribution of the $\mathrm{CO}(2-1)$ line to 
Table 3. Flux densities of NGC 4214 for an aperture of $5^{\prime}$ radius centred at RA $=12 \mathrm{~h} 15 \mathrm{~m} 39.1 \mathrm{~s} \mathrm{Dec}=+36^{\circ} 19^{\prime} 34^{\prime \prime}$ (J (J2000) (see Fig. 2).

\begin{tabular}{lccccc}
\hline \hline Band & $\lambda_{0}(\mu \mathrm{m})$ & $F_{\text {DISK }}^{\text {sub }}(\mathrm{Jy})$ & $C_{\text {color }}$ & $F_{\text {DISK }}(\mathrm{Jy})$ & $F_{\text {DIFF }}(\mathrm{Jy})$ \\
\hline IRAC8 & 7.872 & 0.678 & 0.590 & $1.149 \pm 0.115$ & $0.695_{-0.090}^{+0.075}$ \\
MIPS24 & 23.680 & 2.050 & 0.986 & $2.080 \pm 0.208$ & $0.625_{-0.124}^{+0.104}$ \\
MIPS70 & 71.440 & 23.945 & 0.893 & $26.814 \pm 5.363$ & $13.417_{-4.034}^{+3.082}$ \\
PACS70 & 70.000 & 24.209 & 0.982 & $24.652 \pm 2.466$ & $11.703_{-1.672}^{+1.393}$ \\
PACS100 & 100.000 & 35.309 & 0.985 & $35.847 \pm 3.585$ & $21.128_{-2.735}^{+2.279}$ \\
PACS160 & 160.000 & 34.097 & 1.010 & $33.759 \pm 3.376$ & $22.967_{-2.856}^{+2.380}$ \\
MIPS160 & 155.900 & 39.016 & 0.971 & $38.574 \pm 7.715$ & $27.287_{-6.042}^{+5.605}$ \\
SPIRE250 & 250.000 & 18.888 & 0.992 & $19.033 \pm 2.855$ & $14.594_{-2.645}^{+2.204}$ \\
Planck350 & 350.000 & 9.546 & 0.986 & $9.682 \pm 0.988$ & $7.933_{-0.870}^{+0.797}$ \\
SPIRE350 & 363.000 & 10.059 & 0.999 & $10.068 \pm 1.510$ & $8.453_{-1.321}^{+1.281}$ \\
SPIRE500 & 517.000 & 4.497 & 1.025 & $4.387 \pm 0.659$ & $3.848_{-0.590}^{+0.580}$ \\
Planck550 & 550.000 & 3.635 & 0.921 & $3.947 \pm 1.318$ & $3.510_{-1.177}^{+1.173}$ \\
Planck850 & 850.000 & 0.946 & 0.887 & $1.066 \pm 0.264$ & $0.958_{-0.232}^{+0.231}$ \\
MAMBO* & 1200.000 & 0.350 & 1.000 & $0.350 \pm 0.106$ & $0.288_{-0.105}^{+0.087}$ \\
\hline
\end{tabular}

Notes. $F_{\mathrm{DISK}}^{\mathrm{sub}}$ is the measured, background-subtracted flux, $F_{\mathrm{DISK}}$ is the final flux of the entire galaxy, after applying colour correction, and $F_{\mathrm{DIFF}}$ is the flux of the diffuse emission obtained as $F_{\mathrm{DISK}}-\left(F_{\mathrm{NW}}+F_{\mathrm{SE}}\right)$. The lower error of $F_{\mathrm{DIFF}}$ includes the contribution of smaller and less intense SF regions in the disk (see Sect. 6). ${ }^{(*)}$ The contribution from thermal radio emission was not subtracted.

the total measured flux at $1.2 \mathrm{~mm}$ of the NW and SE complex is then $0.87 \%$ and $1.27 \%$, respectively. For other bands, the contamination by higher $\mathrm{CO}$ transitions is more than one order of magnitude less.

PACS spectroscopic maps of atomic FIR lines ([OI] 63, [OIII] 88, [NII] 122, [OI] 146, [CII] 158, and [NII] $205 \mu \mathrm{m}$ ) of NGC 4214 were presented by Cormier et al. (2010). With a field of view of $1.6^{\prime} \times 1.6^{\prime}$, only the central part of the galaxy is observed. However, this region completely covers the main SF complexes NW and SE where the major part of the line emission originates. Therefore, we do not expect to severely underestimate the total emission of these lines. [OI] 63 and [OIII] $88 \mu \mathrm{m}$ fall into the bandpass of the MIPS 70 and PACS 70 filter. The luminosities reported by Cormier et al. (2010) are $0.89 \times 10^{6} L_{\odot}$ for [OI] $63 \mu \mathrm{m}$ and $1.99 \times 10^{6} L_{\odot}$ for [OIII] $88 \mu \mathrm{m}$. The sum of these values corresponds to $1.11 \%(1.33 \%)$ of the total luminosity measured in the PACS 70 (MIPS 70) band. [OIII] $88 \mu \mathrm{m}$ fall into the bandpass of the PACS 100 filter. Its luminosity corresponds to $1.73 \%$ of the total luminosity measured in the PACS 100 band. [OI] 146 and [CII] $158 \mu \mathrm{m}$ fall into the bandpass of the MIPS 160 and PACS 160 filter. The reported luminosities for [OI] 146 and [CII] $\mu \mathrm{m}$ lines are $0.05 \times 10^{6} L_{\odot}$ and $2 \times 10^{6} L_{\odot}$, respectively, which together represent $2.88 \%(4.26 \%)$ of the total luminosity measured in the PACS 160 (MIPS 160) band.

The small contribution from the atomic and molecular lines to the FIR/submm band meant we did not apply any decontamination to our measurements except for the PACS 160 and MIPS 160 bands. These have been included in Tables 1-3.

\subsection{Error handling}

In our error analysis we took three types of error into account: (i) calibration, $\Delta_{\text {cal }}$, for which we adopted the values mentioned in Sect. 2; (ii) measurement error due to background fluctuations, $\Delta_{\text {back }}$; and (iii) an estimate of the error due to the uncertainty in the aperture size of regions SE and NW, $\Delta_{\text {apert }}$ (this error was not relevant for the total emission). We neglected the uncertainties introduced by the colour and aperture corrections.

The error due to the background fluctuations was calculated by assuming that each pixel within the aperture has an error given by the standard deviation of the background noise, $\sigma_{\text {back }}$. In addition, we had to take the error of the background into account which was subtracted within an aperture of $N_{\text {apert }}$ pixels. This error is $\sigma_{\text {back }} N_{\text {apert }} / \sqrt{N_{\text {back }}}$, where $N_{\text {back }}$ is the number of pixels used to compute the level of background. This gives (see also Dale et al. 2012) a total error for the background subtracted flux of

$\Delta_{\text {back }}=\sigma_{\text {back }} \sqrt{N_{\text {apert }}+\frac{N_{\text {apert }}^{2}}{N_{\text {back }}}}$.

We estimated the error $\Delta_{\text {apert }}$ by changing the aperture size for the regions NW and SE by $\pm 1^{\prime \prime}$. We obtained changes in the integrated fluxes between 4 and $8 \%$ for the different bands. We conservatively adopted an error of $\Delta_{\text {apert }}=8 \%$ for all bands.

The final error for the flux is the quadratic sum of $\Delta_{\text {cal }}, \Delta_{\text {back }}$, and $\Delta_{\text {aper. }}$ This is listed in Tables 1-3 for the fluxes in each band. The dominant error sources were $\Delta_{\text {cal }}$ and $\Delta_{\text {aper }}$, whereas $\Delta_{\text {back }}$ was found to be negligible for most wavelengths.

\section{Models for the dust emission}

We analyse the full UV to FIR/submm SED of the different emission components of the galaxy using the radiation transfer model of Popescu et al. (2011), which self-consistently treats the dust emission from diffuse and SF complexes components, considering the illumination of diffuse dust both by the distributed stellar populations and by the escaping light from the HII regions. While maintaining consistency within the framework of Popescu et al. (2011) model, we use the model of Groves et al. (2008) to provide a detailed description of the dust emission from the two central SF complexes NW and SE. In the following sections we present a brief description of the physics and parameters of these models. 


\subsection{The model of Popescu et al. (2011)}

Popescu et al. (2011) present a self-consistent model based on full radiative transfer calculations of the propagation of starlight in disk galaxies. To approximate the large-scale geometry of the galaxy (see Fig. 1 in Popescu et al. 2011), they use two separate components: (i) an old component consisting of an old stellar disk, an old stellar bulge, and a dust disk; and (ii) a young component consisting of a young stellar disk and a dust disk. The young component is introduced to mimic the more complex distribution of young stars and diffuse dust associated with the spiral arms. Apart from the diffuse component, the model includes a clumpy component, consisting of the parent molecular clouds of massive stars. The input parameters of this model are

- the total central face-on $B$-band opacity, $\tau_{\mathrm{B}}^{\mathrm{f}}$, which is the sum of the central face-on, $B$-band opacities of the young and old dust disks;

- the SF rate, SFR;

- the clumpiness factor $F$, which can be physically identified with the luminosity-weighted mean fraction of directions from the massive stars, averaged over the lifetime of the stars, which intersects the birth-cloud. The clumpiness factor $F$ is linked to the fraction of photons that escapes $\left(f_{\text {esc }}\right)$ from the SF regions into the diffuse medium as $F=1-f_{\text {esc }}$;

- the normalized luminosity of the old stellar disk, old;

- the bulge-to-disk ratio, $B / D$, which determines the bulge contribution to the old stellar radiation field;

- the radial scale length of the old stellar disk, $h_{\mathrm{s}}$, which defines the size of the galaxy. All other spatial scales in the galaxy (scalelength of the young stellar population, scalelength of the dust and vertical scaleheights for the different components) have a constant ratio with $h_{\mathrm{s}}$ (see Table E. 1 in Popescu et al. 2011);

- the inclination angle, $i$.

From the primary parameters $S F R$ and $F$, Popescu et al. (2011) define the SFR powering the diffuse emission, $S F R^{\prime}$, as follows (Eq. (45) in Popescu et al. 2011):

$S F R^{\prime}=S F R \times(1-F)$.

The library of diffuse SEDs of Popescu et al. (2011) contains results for a four-dimensional parameter space spanned by $\tau_{\mathrm{B}}^{\mathrm{f}}$, $S F R^{\prime}$, old, and $B / D$. The diffuse component is calculated as an extrinsic quantity corresponding to a reference size (corresponding to a reference scalelength). To scale the intensity of the ISRF heating the diffuse dust in a galaxy, the parameters $S F R^{\prime}$ and old must be scaled to the reference size by comparing the scalelengths (Eq. (D.3) in Popescu et al. 2011):

$S F R^{\text {model }}=S F R^{\prime} \times\left(\frac{h_{\mathrm{s}}^{\mathrm{ref}}(B)}{h_{\mathrm{s}}(B)}\right)^{2}$
$o l d^{\text {model }}=\operatorname{old} \times\left(\frac{h_{\mathrm{s}}^{\mathrm{ref}}(B)}{h_{\mathrm{s}}(B)}\right)^{2}$,

where $h_{\mathrm{s}}^{\mathrm{ref}}(B)=5670 \mathrm{pc}$ and $h_{\mathrm{s}}(B)$ are the reference $B$-band scalelength and the $B$-band scalelength of the galaxy under study, respectively. We note here that $S F R^{\text {model }}$ and old $^{\text {model }}$ are only internal parameters that allow us to interface with the library of models. Because of this all the results in this paper are presented only in terms of the $S F R$ and old, the real parameters of the galaxy under study.

An additional scaling is required to set the flux levels of the SEDs from the library to the observed SED of our galaxy.
Thus, the SED that represents our galaxy, $F_{\lambda}^{\mathrm{d}}$, is determined as (Eq. (D.2) in Popescu et al. 2011):

$F_{\lambda}^{\mathrm{d}}=\left(\frac{h_{\mathrm{s}}(B)}{h_{\mathrm{s}}^{\mathrm{ref}}(B)}\right)^{2} \times F_{\lambda}^{\mathrm{d} \text {,model }}\left(B / D, \tau_{\mathrm{B}}^{f}, S F R^{\text {model }}\right.$, old $\left.^{\text {model }}\right)$.

We would like to stress that in these models the absolute flux level of the predicted dust SED is fixed by the input parameters.

The models of Popescu et al. (2011) have been designed to provide the formalism for fitting the integrated emission of galaxies, since resolved information on the dust emission is not available for most galaxies. In particular, dust emission SEDs of individual starforming complexes cannot be derived in most cases. Consequently, the model of Popescu et al. (2011) provides an average template SED for the dust emission of the ensemble of the HII regions. This template has been empirically calibrated on data of a representative sample of prominent star forming complexes in our Galaxy, as fitted using the model of Groves et al. (2008). In contrast, in this study of NGC 4214, we have resolved information on the central star forming complexes, and can therefore replace the average template with a detailed modelling of these complexes using the model of Groves et al. (2008) directly, while still retaining the general framework of the model of Popescu et al. (2011), when calculating the attenuation of stellar light in the clumpy component. This allows us to reach a selfconsistent treatment of the diffuse and clumpy component, mediated by the fact that the two models used here have a common parameter, the $F$ factor.

\subsection{The model of Groves et al. (2008)}

The Groves et al. (2008) model describes the luminosity evolution of a star cluster of mass $M_{\mathrm{cl}}$, and incorporates the expansion of the HII region and PDR due to the mechanical energy input of stars and SNe. The dust emission from the HII region and the surrounding PDR is calculated from radiation transfer. The main input parameters of the model are

- the metallicity of the star cluster, $Z$, in units of the solar metallicity $Z_{\odot}$ (Asplund et al. 2005). The metallicities used in the model are restricted to the Small Magellanic Could (SMC) and LMC metallicities. This limitation is introduced by the stellar population model (Smith et al. 2002);

- the age of the star cluster, $t$;

- the ambient pressure, $\log \left(p_{0} / k\right)$, which controls the rate of the expansion of the HII region and PDR due to the mechanical energy input of stars and SNe, and the size. Therefore $\log \left(p_{0} / k\right)$ determines the dust temperature for a given star cluster mass and age;

- the compactness parameter, $\log (C)$, which parametrizes the heating capacity of the star cluster and depends on $M_{\mathrm{cl}}$ and $\log \left(p_{0} / k\right)$

- the covering factor, $f_{\text {cov }}$, which represents the fraction of the surface of the HII region covered by the PDR. This is the same parameter as the $\mathrm{F}$ factor in the model of Popescu et al. (2011);

- the hydrogen column density of the PDR, $N_{\mathrm{HI}}^{\mathrm{PDR}}$.

At a given age, the model self-consistently calculates the luminosity distribution of the star cluster and the radius of the inner edge of the PDR and computes the emergent SED for the dust in the HII region without considering the PDR, $F_{\lambda}^{\mathrm{HII}}$, and for the dust from the HII region, which is completely covered by a PDR, $F_{\lambda}^{\mathrm{HII}+\mathrm{PDR}}$. The total emergent SED from the SF region, which is 
partially covered by the PDR, $F_{\lambda}^{\mathrm{SF}}$, is then given by

$F_{\lambda}^{\mathrm{SF}}=\left(1-f_{\mathrm{cov}}\right) \times F_{\lambda}^{\mathrm{HII}}+f_{\mathrm{cov}} \times F_{\lambda}^{\mathrm{HII}+\mathrm{PDR}}$.

\section{Constraints on the input parameters}

The large amount of ancillary data covering a wide wavelength range, as well as the result of previous studies from the literature, allow us to determine or at least constrain most of the input parameters for both models.

\subsection{Input parameters for the model of Groves et al. (2008)}

The two main SF complexes NW and SE are formed by an ensemble of smaller HII regions. Ideally, all these smaller knots of SF should be modelled separately. However, IR/submm observations lack the angular resolution to perform such a detailed analysis. Due to this limitation, for both complexes we adopted parameter ranges that are wide enough to enclose the values of their individual knots. With the only exception of $N_{\mathrm{HI}}^{\mathrm{PDR}}$, for which we adopted the value $10^{22} \mathrm{~cm}^{-2}$, which is typical of molecular clouds in our own galaxy, the input parameters of the model of Groves et al. (2008) were observationally constrained:

(i) Metallicity: the metallicities of both SF regions have been measured by Kobulnicky \& Skillman (1996), who found values of $12+\log (\mathrm{O} / \mathrm{H})=8.17 \pm 0.02$ for the NW and $8.27 \pm 0.02$ for the $\mathrm{SE}$ region. With the solar abundance $12+\log (\mathrm{O} / \mathrm{H})=8.66$ (Asplund et al. 2005), as used in Groves et al. (2008), this gives $Z=0.32 Z_{\odot}$ for $\mathrm{NW}$ and $Z=0.41 Z_{\odot}$ for SE. We used a metallicity of $Z=0.4 Z_{\odot}$ and also tested $Z=0.2 Z_{\odot}$, which yielded a similar result (no template for $Z=0.3 Z_{\odot}$ is available in Groves et al. 2008).

(ii) Age: the age of the star clusters of NGC 4214 have been extensively studied by different authors. Leitherer et al. (1996) presented HST Faint Object Spectrograph (FOS) ultraviolet spectra covering the main star cluster of the NW complex. Using spectral synthesis modelling, Leitherer et al. (1996) reported an age of 4-5 Myr for this stellar cluster. Maíz-Apellániz et al. (1998) obtained optical long-slit spectra of NGC 4214 with the ISIS spectrograph of the William Herschel Telescope. Their bidimensional spectra covered both SF complexes. From the comparison of different observationally determined parameters (equivalent width of $\mathrm{H} \beta$, Wolf-Rayet population, effective temperature and UV absorption lines) to synthesis models, they determined ages of $3 \pm 1$ Myr for both regions. MacKenty et al. (2000) mapped the central part of NGC 4214 with the HST WFPC2. From the $\mathrm{H} \alpha$ equivalent width, these authors determined an average age of 3.0-4.0 Myr for the NW and 2.5-3.0 Myr for the SE region. More recently, Úbeda et al. (2007) have also used the WFPC2 data set to determine the ages of the star clusters by a likelihood-maximization technique from the photometric colours. They determined an age of $5 \mathrm{Myr}$ for the star clusters within the NW complex and 2-4 Myr for the SE complex. Based on the dispersion found by these studies, we adopted ages of 3-5 Myr for the NW and 2-4 Myr for the SE complex.

(iii) Ambient pressure and compactness: $\log \left(p_{0} / k\right)$ and $\log (C)$ were determined by comparing the expected and the observed radii of the individual HII regions as a function of the age. For a cluster of a given age it is possible to find different combinations of $\log \left(p_{0} / k\right)$ and $\log (C)$ that provide the observed radius of the expansion bubble. However, this degeneracy can be avoided when the mass of the star cluster is known, since both parameters $\log \left(p_{0} / k\right)$ and $\log (C)$ are related by the equation

$\log \left(\frac{M_{\mathrm{cl}}}{M_{\odot}}\right)=\frac{5}{3} \times \log (C)-\frac{2}{3} \times \log \left(\frac{p_{0} / k}{\mathrm{~cm}^{-3} \mathrm{~K}}\right)$.

From the values of the masses reported by Úbeda et al. (2007) we found that for the NW complex the value of $\log \left(p_{0} / k\right)$ ranges from 7.0 to 8.0 and $\log (C)$ ranges from 5.5 to 6.5 depending on the HII region considered. For the case of the SE complex, we found that $\log \left(p_{0} / k\right)$ ranges from 6.0 to 7.0 and that $\log (C)$ ranges from 4.5 to 5.5 . We used these intervals to constrain both parameters.

(iv) Covering factor: we assumed that the PDR consists of optically thick, homogeneously distributed clouds that surround the star cluster, leaving a fraction uncovered so that the light can escape unattenuated from these "holes". To a good approximation, the intrinsic luminosity of the central star cluster is the sum of the observed luminosities of the stars, $L_{\text {star }}$, and the luminosity re-emitted by the dust, $L_{\text {dust }}$. The covering factor is then $f_{\text {cov }}=L_{\text {dust }} /\left(L_{\text {dust }}+L_{\text {star }}\right)$. We obtained $L_{\text {dust }}$ by integrating the best-fit model template (see Sect. 6) from $3 \mu \mathrm{m}$ to $1.5 \mathrm{~mm}$. To calculate $L_{\text {star }}$ we integrated the de-attenuated fluxes measured in our apertures for the SF regions from GALEX FUV to IRAC $3 \mu \mathrm{m}$. The exact value of the opacity caused by the diffuse dust layer in front of the SF regions is not known. For this reason, we carried out two different estimates, the first based on the lowest and the second on the highest realistic opacity. (i) We adopted the foreground opacity of $\tau_{\mathrm{V}}=0.35$ (Úbeda et al. 2007) measured locally in front of the main stellar cluster of the NW region and assumed that this value is representative of both SF regions. The stars in the NW region seem to have evacuated most of the surrounding material associated with the HII region so that this value is expected to be lower than the average disk opacity in this area. We obtained $f_{\text {cov }}^{\mathrm{NW}}=0.45$ and $f_{\text {cov }}^{\mathrm{SE}}=0.65$ for the NW and SE regions, respectively. (ii) We used the value of $\tau_{\mathrm{B}}^{\mathrm{f}}=2.0$ which is the best-fit value derived from our modelling of the diffuse emission (see Sect. 6). Assuming that the regions are in mid-plane and using the inclination angle $i=44^{\circ}$ (Walter et al. 2008), the opacity in front of the SF complexes is calculated as $0.5 \times \tau_{\mathrm{B}}^{\mathrm{f}} / \cos (i)=1.4$. In this case, we obtained $f_{\text {cov }}^{\mathrm{NW}}=0.20$ and $f_{\text {cov }}^{\mathrm{SE}}=0.30$. Thus, we obtain estimates for the covering factor of $f_{\mathrm{cov}}^{\mathrm{NW}}=0.20-0.45$ and $f_{\mathrm{cov}}^{\mathrm{SE}}=0.30-0.65$ for both regions.

\subsection{Input parameters for the model of Popescu et al. (2011)}

In the case of the model of Popescu et al. (2011) the parameters that we could determine observationally are:

(i) Bulge-to-disk ratio: since no bulge is visible in the late-type galaxy NGC 4214 we set $B / D=0$.

(ii) Inclination angle: we fix $i=44^{\circ}$ following Walter et al. (2008).

(iii) Scalelength: we determined the radial stellar scalelength in the $B$-band from a SLOAN g-band image. First, we removed the background of the image by calculating the average value of a wide set of small circular apertures placed strategically outside the disk of NGC 4214. Then, we removed the contamination from foreground stars and HII regions, including the central regions SE and NW. We used the IRAF task ellipse (STSDAS package) with steps of $20^{\prime \prime}$ to determine the isophotes. For all 
I. Hermelo et al.: The dust SED of dwarf galaxies. I.

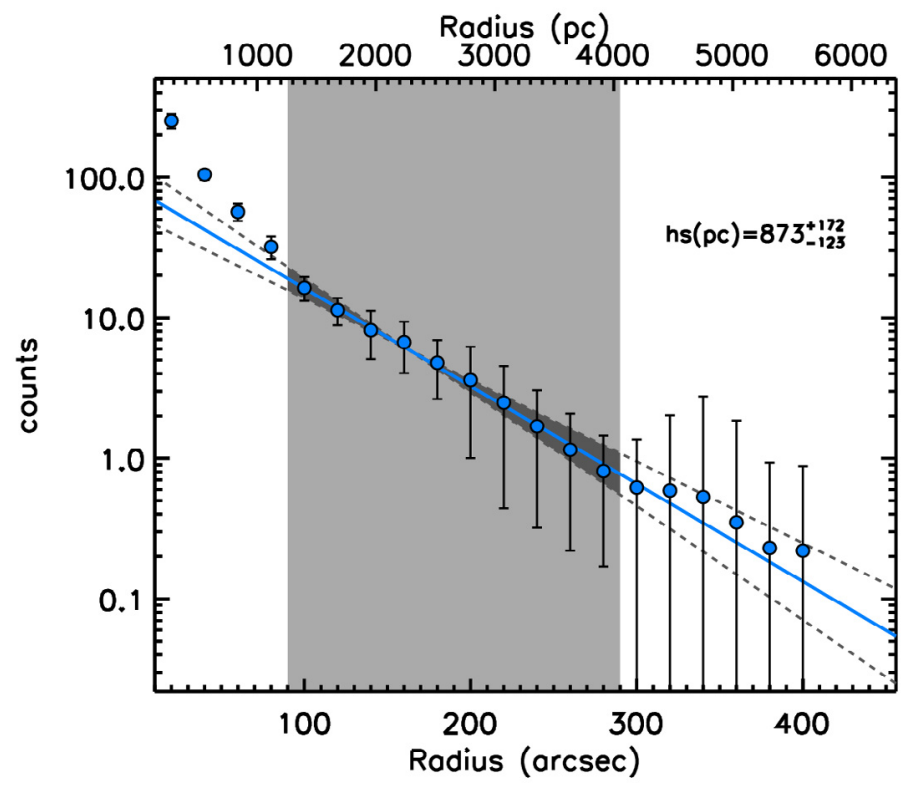

Fig. 3. $B$-band scalelength of the stellar disk of NGC 4214. The fit (solid line) was achieved using the isophotes in the radial range marked by the grey-shaded area. The uncertainty in the slope is represented by the dark grey area and the dark grey dashed lines.

the isophotes beyond $100^{\prime \prime}$ from the centre, the PA and the ellipticity were fixed. Finally, we fitted an exponential function to the surface brightness profile determined with the mean values in each isophotal ellipse. We considered the ellipses whose mean values and the corresponding uncertainties were above the background level.

We found that it is not possible to fit the profile for the whole disk with a single exponential function. For this reason we decided to calculate the scalelength separately for the inner and the outer parts of the disk (see Fig. 3). We visually determined the change of slope to take place at a radius of $\sim 90^{\prime \prime}$. The profile of the inner part of the disk (excluding the HII regions NW and $\mathrm{SE})$ can be well fitted with a scalelength of $\sim 450 \mathrm{pc}$. For the outer part we determined a scalelength of $873_{-123}^{+172} \mathrm{pc}$.

(iv) Clumpiness: as a first estimate of the clumpiness factor $F$ we used the mean value from the upper and the lower limits obtained for the two SF regions, i.e., $F=0.40 \pm 0.20$. This parameter is only used for modelling the total integrated UV/optical/FIR emission described in Sect. 6.3. In Sect. 6.2 we present an additional global estimate of $F$.

( $v$ ) old: we derived this parameter as old $=0.009$ by assuming that all the luminosity in the $J, H$, and $K$ bands (see Table 4) corresponds to the old stellar population. We integrated the luminosity of NGC 4214 in the $J, H$, and $K$ bands and derived old as the ratio of this luminosity and the integrated luminosity of the model galaxy (Table E2 in Popescu et al. 2011).

\section{Results}

In a first step we separately fitted the emission from the SF regions and from diffuse dust. In a second step we self-consistently combined the emission from the SF regions with that from the diffuse dust to fit the total emission of the galaxy.

The emission from the NW and SE regions has been directly measured from the maps as described in Sect. 3. The diffuse emission has been calculated as the difference between the total
Table 4. Total stellar flux densities of NGC 4214.

\begin{tabular}{lccc}
\hline \hline Band & $\lambda_{0}(\mu \mathrm{m})$ & $F_{\text {DISK }}(\mathrm{Jy})$ & Reference \\
\hline FUV & 0.154 & $0.072 \pm 0.007$ & 1 \\
NUV & 0.232 & $0.091 \pm 0.009$ & 1 \\
$B$ & 0.445 & $0.354 \pm 0.056$ & 2 \\
$V$ & 0.551 & $0.446 \pm 0.064$ & 2 \\
$J$ & 1.220 & $0.520 \pm 0.014$ & 3 \\
$H$ & 1.630 & $0.614 \pm 0.023$ & 3 \\
$K$ & 2.200 & $0.458 \pm 0.022$ & 3 \\
\hline
\end{tabular}

References. (1) This work, (2) de Vaucouleurs et al. (1991b), (3) Jarrett et al. (2003).

emission and the sum of the emissions from the two SF complexes. For the data points where no direct measurement was available, the best-fit model value (see below) was taken. The values for the diffuse dust emission are listed in Table 3.

To take the emission from smaller and less intense $\mathrm{SF}$ regions in the disk of NGC 4214 into account, we estimated their contribution from the $\mathrm{H} \alpha$ emission of the ten brightest secondary SF regions, which corresponds to $18 \%$ of the $\mathrm{H} \alpha$ emission of SE + NW. We assume that the shape of the dust SED from these smaller SF regions is the same as the sum of the SEDs of SE + $\mathrm{NW}$, and we include their contribution in the calculation of the lower error range of the fluxes of the diffuse dust component in each band (see Table 3 ). The smaller HII regions only have a noticeable effect on the emission at $24 \mu \mathrm{m}$ and $70 \mu \mathrm{m}$.

\subsection{Best fits for the SF regions}

The best-fit models for the NW and SE complexes are shown in Figs. 4 and 5, respectively. We obtained a good fit for all our observed photometric points longwards of $10 \mu \mathrm{m}$. For a metallicity of $Z=0.4 Z_{\odot}$, the best-fit models of the two complexes correspond to the parameters $t=4.0 \mathrm{Myr}, \log (C)=5.0, \log \left(p_{0} / k\right)=8$, and $f_{\text {cov }}=0.30$ for both regions. With the exception of $\log (C)$ of the NW region and $\log \left(p_{0} / k\right)$ of the SE region, all the parameters fall within our parameter ranges constrained from the observations (see Sect. 5.1). For a metallicity of $Z=0.2 Z_{\odot}$, the best fit parameters are, in the case of the NW region, $t=5.0 \mathrm{Myr}$, $\log (C)=5.0, \log \left(p_{0} / k\right)=7$, and $f_{\text {cov }}=0.30$, and in the case of the SE region, $t=3.5 \mathrm{Myr}, \log (C)=4.5, \log \left(p_{0} / k\right)=8$, and $f_{\text {cov }}=0.60$. The value for $\log (C)$ in $\mathrm{NW}$ is slightly lower, and $\log \left(p_{0} / k\right)$ in SE slightly higher, but the rest of the parameters are within the observed ranges.

We note that the IRAC $8 \mu \mathrm{m}$ data point was excluded from our fitting procedure. The reason is that both models considerably underestimate the emission at $8 \mu \mathrm{m}$. Specifically, from our best-fit models for $Z=0.2 Z_{\odot}$ we find that the observed fluxes are larger than the model values by a factor of 3.8 for $\mathrm{NW}$ and 4.0 for SE. In the case of the best-fit models of $Z=0.4 Z_{\odot}$ the discrepancy decreases to a factor of 3.1 for NW and 2.9 for SE. A complete discussion of the IRAC $8 \mu \mathrm{m}$ discrepancy is given in Sect. 7.

\subsection{Best fit for the diffuse emission}

We searched the library of the diffuse dust SEDs of Popescu et al. (2011) for the best fit to the data in the MIR/submm range, leaving $\tau_{\mathrm{B}}^{\mathrm{f}}$ and $S F R^{\prime}$ as free parameters and keeping old fixed to the value 0.009 . Figure 6 shows the best fit obtained for our measured value $h_{\mathrm{s}}=873 \mathrm{pc}$, where we determined as best-fit 

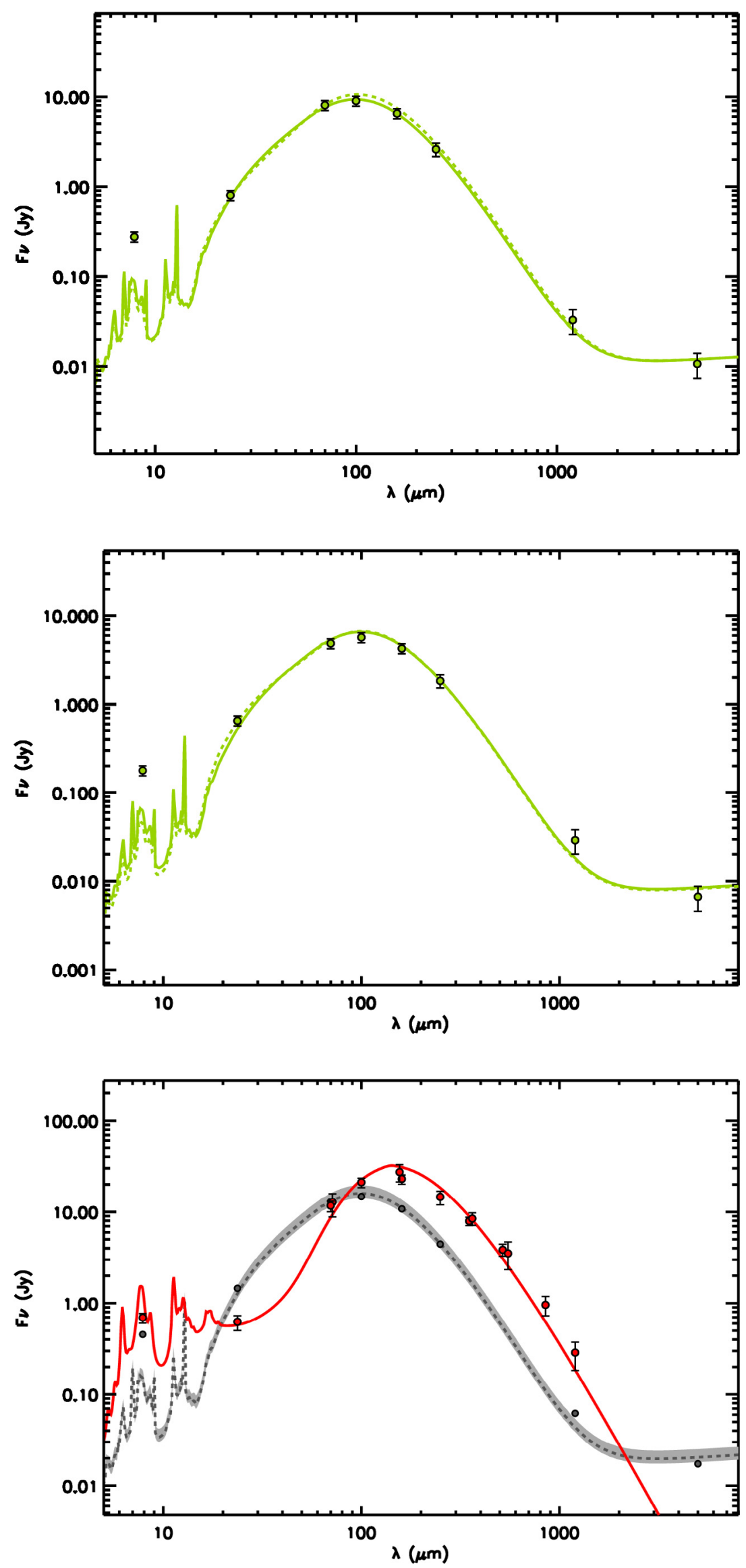

Fig. 4. Best-fit models to the NW complex for $Z=$ $0.4 Z_{\odot}$ (solid line) and $Z=0.2 Z_{\odot}$ (dashed line). The values of the reduced $\chi^{2}$ (neglecting the IRAC $8 \mu \mathrm{m}$ point) are 0.25 for $Z=0.4 Z_{\odot}$ and 0.75 for $0.2 Z_{\odot}$.

Fig. 5. Best-fit model to the SE complex for $Z=0.4 Z_{\odot}$ (solid line) and $Z=0.2 Z_{\odot}$ (dashed line). The values of the reduced $\chi^{2}$ (neglecting the IRAC $8 \mu \mathrm{m}$ point) are 1.17 for $Z=0.4 Z_{\odot}$ and 0.89 for $0.2 Z_{\odot}$.

Fig. 6. Fit to the diffuse emission of NGC 4214 keeping fixed old $=0.009$ and leaving $\tau_{\mathrm{B}}^{\mathrm{f}}$ and $S F R^{\prime}$ as free parameters. The solid red line represents the best fit to the MIR/submm SED for the measured value of the scalelength $h_{\mathrm{s}}=873 \mathrm{pc}$, obtained for $\tau_{\mathrm{B}}^{\mathrm{f}}=2.0$, and $S F R^{\prime}=0.059 M_{\odot} \mathrm{yr}^{-1}$. The dark grey dashed line corresponds to the sum of the best-fit models of the HII regions presented in Figs. 4 and 5, and the greyfilled circles correspond to the sum of the photometric points of NW and SE regions. The light grey area represents the uncertainty in the estimation of the total emission of the HII regions. The value of the reduced $\chi^{2}$ (neglecting the IRAC $8 \mu \mathrm{m}$ and MAMBO points) is 1.82 . 
values $\tau_{\mathrm{B}}^{\mathrm{f}}=2$ and $S F R^{\prime}=0.059 M_{\odot} \mathrm{yr}^{-1}$. Neither the data point at $8 \mu \mathrm{m}$ nor the one at $1.2 \mathrm{~mm}$ were taken into account in the $\chi^{2}$ fitting procedure, the latter due to the observational limitations discussed in Sect. 3.1.

The model fits the data points in general very well. The largest discrepancy occurs at $8 \mu \mathrm{m}$ where the model overpredicts the observations by a factor of 1.9. We discuss this further in Sect. 7. At $160 \mu \mathrm{m}$ there is a discrepancy with the PACS value, which is overpredicted by $34 \%$ by the model, but the model prediction agrees within the errors with the MIPS data point at the same wavelength. The model overpredicts the SPIRE $250 \mu \mathrm{m}$ point by $24 \%$ and underpredicts the $850 \mu \mathrm{m}$ point by $34 \%$. For the other data points, the model predictions agree within the error bars.

It is instructive to compare this value of $S F R^{\prime}$ derived from the fit to the diffuse component of the dust emission, with the value for $S F R^{\prime}$ derived from our measurement of the spatially integrated flux density in the UV-to-blue band, which we call, for clarity, $S F R^{\prime}{ }_{\text {UV }}$ in the following. Applying the attenuation corrections due to diffuse dust, as tabulated in Popescu et al. (2011) for $\tau_{\mathrm{B}}^{\mathrm{f}}=2$ and an inclination of $44^{\circ}$, we derive a value of $L_{\mathrm{UV}}=$ $3.70 \times 10^{35} \mathrm{~W}$. We then determine $S F R^{\prime}{ }_{\mathrm{UV}}$ following Eq. (17) in Popescu et al. (2011) as $S F R_{\mathrm{UV}}^{\prime}=L_{\mathrm{UV}} / L_{\text {unit,UV }}^{\text {young }}$, where $L_{\text {unit,UV }}^{\text {young }}$ is the normalization factor. We obtain $S F R_{U V}^{\prime}=0.165 M_{\odot} \mathrm{yr}^{-1}$. This is a factor of 2.8 times higher than the value of $S F R^{\prime}$ derived from fitting the diffuse dust emission. The most straightforward explanation for this discrepancy would be that the true value for the disk scalelength $h_{\mathrm{s}}$ is higher than the adopted of $873 \mathrm{pc}$. This would favour a disk with lower dust surface densities, leading to a higher value of $S F R^{\prime}$, which is needed to account for the observed amplitude and colour of the diffuse dust emission. If we adopt for the scalelength the maximum allowed by the $B$-band images, $h_{\mathrm{s}}=1045 \mathrm{pc}$, we obtain a best fit to the diffuse dust emission SED of $\tau_{\mathrm{B}}^{\mathrm{f}}=1.2$ and $S F R^{\prime}=0.088 M_{\odot} \mathrm{yr}^{-1}$. At the same time, this reduction in $\tau_{\mathrm{B}}^{\mathrm{f}}$ means that the deattenuated, integrated UV luminosity, $L_{\mathrm{UV}}$, is now lower, yielding $S F R_{U V}^{\prime}=0.147 M_{\odot} \mathrm{yr}^{-1}$ which is only a factor of 1.67 greater than the value of $S F R^{\prime}$ derived from the diffuse dust emission. A complete discussion of this discrepancy is given in Sect. 7.

As a further consistency check, we can use the value of $S F R^{\prime}$ derived from the diffuse dust emission to make a different, global estimate of the fraction of the UV radiation escaping from the SF regions, $f_{\text {esc }}$, and the corresponding clumpiness factor, $F$, and compare it with the corresponding value already derived in Sect. 5 from analysis of the direct and dust-re-radiated UV light seen from the spatially resolved SF regions. For this, we have to assume that the effective SFR powering the diffuse dust, $S F R^{\prime}$, is due to radiation escaping from the regions NW and SE. The total effective SFR produced by $\mathrm{NW}+\mathrm{SE}$ is then $S F R^{\prime}+S F R_{\mathrm{loc}}$ where $S F R_{\text {loc }}$ is the effective SFR needed to power the dust emission from the SF regions. We calculate $S F R_{\text {loc }}$ by integrating the dust SED from the SF regions over the entire wavelength range, obtaining $L_{\text {dust }}$, and assuming that this luminosity is equivalent to the same amount of UV radiation absorbed by the dust locally. We then determine $S F R_{\text {loc }}$ in an equivalent way to $S F R_{\mathrm{UV}}^{\prime}$ as described above, as $S F R_{\mathrm{loc}}=L_{\mathrm{dust}} / L_{\text {unit,UV }}^{\text {young }}$. We obtain $L_{\text {dust }}=9.06 \times 10^{34} \mathrm{~W}$, which gives $S F R_{\text {loc }}=0.040 M_{\odot} \mathrm{yr}^{-1}$. With $S F R^{\prime}=0.059 M_{\odot} \mathrm{yr}^{-1}$, derived from our best fit for the diffuse emission, we then obtain $f_{\mathrm{esc}}=S F R^{\prime} /\left(S F R^{\prime}+S F R_{\mathrm{loc}}\right)=60 \%$ of the UV radiation of the SF region is required to escape from the SF complexes in order to heat the diffuse dust. This escape fraction corresponds to a $f_{\text {cov }}=F=1-0.60=0.40$, which is the same value as derived in Sect. 5. We thus adopt in the following
$F=0.40 \pm 0.20$ (where the error comes from the range of $f_{\text {cov }}$ derived in Sect. 5.1). From the best-fit value of $S F R^{\prime}$ and $F=0.40$, we can estimate the value of the total SFR of NGC 4214 as $S F R=S F R^{\prime} /(1-F)=0.059 /(1-0.40)=0.098 M_{\odot} \mathrm{yr}^{-1}$.

\subsection{Best fit for the total emission}

In the MIR/FIR/submm part of Fig. 7 we show the fit to the total dust emission, which is the sum of the SEDs from the SF regions and the SED from the diffuse dust obtained in the previous sections. The entire dust SED from $8 \mu \mathrm{m}$ to $850 \mu \mathrm{m}$ can be well fitted.

In the UV/optical/NIR part of Fig. 7 we show the observed UV-optical SED. Following Eq. (C.12) of Popescu et al. (2011), the UV/optical SED of the young stellar disk of NGC 4214 was dereddened using the composite attenuation, $\Delta m_{\lambda}$, which is for the case of old $\sim 0$

$\Delta m_{\lambda}=-2.5 \log \left(1-F f_{\lambda}\right)+\Delta m_{\lambda}{ }^{\text {tdisk }}$

where the first part takes the attenuation in the SF regions and $\Delta m_{\lambda}{ }^{\text {tdisk }}$ the attenuation of the diffuse component into account. The wavelength dependence of the escape fraction, $f_{\lambda}$, is tabulated in Table A.1 in Tuffs et al. (2004). For the old stellar population we used the attenuation correction derived for the old stellar component in the model of Popescu et al. (2011) $\Delta m_{\lambda}=\Delta m_{\lambda}{ }^{\text {disk }}$. For both stellar populations we used the value of $\tau_{\mathrm{B}}^{\mathrm{f}}=2.0$ obtained from our best-fit model and an inclination angle of $i=44^{\circ}$ (Walter et al. 2008). The SED derived in this way represents the total intrinsic stellar SED of NGC 4214.

We now calculate, in a similar way as in Sect. 6.2 for $S F R^{\prime}$, from the intrinsic UV-to-blue luminosity the predicted value of $S F R$, by applying Eq. (17) from Popescu et al. (2011), $S F R=L_{\mathrm{UV}}^{\text {tdisk }} / L_{\text {unit,UV }}^{\text {young }}$. Using $F=0.40$ in Eq. (9), we derive $S F R=0.22 M_{\odot} \mathrm{yr}^{-1}$, which is a factor of 2.24 higher than the value derived from the fitting of the dust SED $(S F R=$ $\left.0.098 M_{\odot} \mathrm{yr}^{-1}\right)$. For $F=0.20$ and $F=0.60$, the highest and lowest values suggested by our data, the corresponding ratios are 2.51 and 2.18 , respectively. If we adopt for the scalelength the maximum value allowed by the data $h_{\mathrm{s}}=1045 \mathrm{pc}$, we obtain the best fit for $\tau_{B}^{f}=1.2$ and $S F R^{\prime}=0.088 M_{\odot} \mathrm{yr}^{-1}$, yielding $S F R=S F R^{\prime} /(1-F)=0.088 /(1-0.40)=0.14 M_{\odot} \mathrm{yr}^{-1}$. In this case, the discrepancy with the value derived from the dereddened UV-optical data, $S F R=0.188 M_{\odot} \mathrm{yr}^{-1}$, decreases to a factor of 1.34 for $F=0.40,1.50$ for $F=0.20$, and 1.27 for $F=0.60$. A complete discussion of this discrepancy is given in Sect. 7.

\subsection{Gas-to-dust mass ratio}

The total diffuse dust mass can be calculated from Eq. (44) of Popescu et al. (2011) $\left(M_{\text {dust }}^{\text {diff }}=\tau_{\mathrm{B}}^{\mathrm{f}} \times h_{\mathrm{s}}^{2} \times 0.99212 \mathrm{pc}^{-2} M_{\odot}\right)$. With our values of $h_{\mathrm{s}}=873 \mathrm{pc}$ and $\tau_{\mathrm{B}}^{\mathrm{f}}=2$ we obtain $M_{\text {dust }}^{\text {diff }}=1.5 \times$ $10^{6} M_{\odot}$. This value is quite robust against changes in parameters. Choosing the largest permitted scalelength, $h_{\mathrm{s}}=1045 \mathrm{pc}$ and the corresponding best fit for the opacity, $\tau_{\mathrm{B}}^{\mathrm{f}}=1.2$, we obtain $M_{\text {dust }}^{\text {diff }}=1.3 \times 10^{6} M_{\odot}$, only $13 \%$ lower. From the modelling of the HII complexes for $Z=0.4 Z_{\odot}$, we derived dust masses of $M_{\text {dust }}^{\mathrm{NW}}=0.79 \times 10^{5} M_{\odot}$ and $M_{\text {dust }}^{\mathrm{SE}}=0.42 \times 10^{5} M_{\odot}$ for the NW and SE complexes, respectively. We therefore derived for NGC 4214 a total mass of dust $M_{\text {dust }}^{\text {total }}=M_{\text {dust }}^{\text {diff }}+M_{\text {dust }}^{\mathrm{NW}}+M_{\text {dust }}^{\mathrm{SE}}=1.62 \times 10^{6} M_{\odot}$.

The total atomic gas mass of NGC 4214 is $M_{\mathrm{HI}}=4.1 \times$ $10^{8} M_{\odot}$ (Walter et al. 2008). NGC 4214 has been mapped in $\mathrm{CO}(2-1)$ as part of the HERACLES survey (Leroy et al. 2009) 


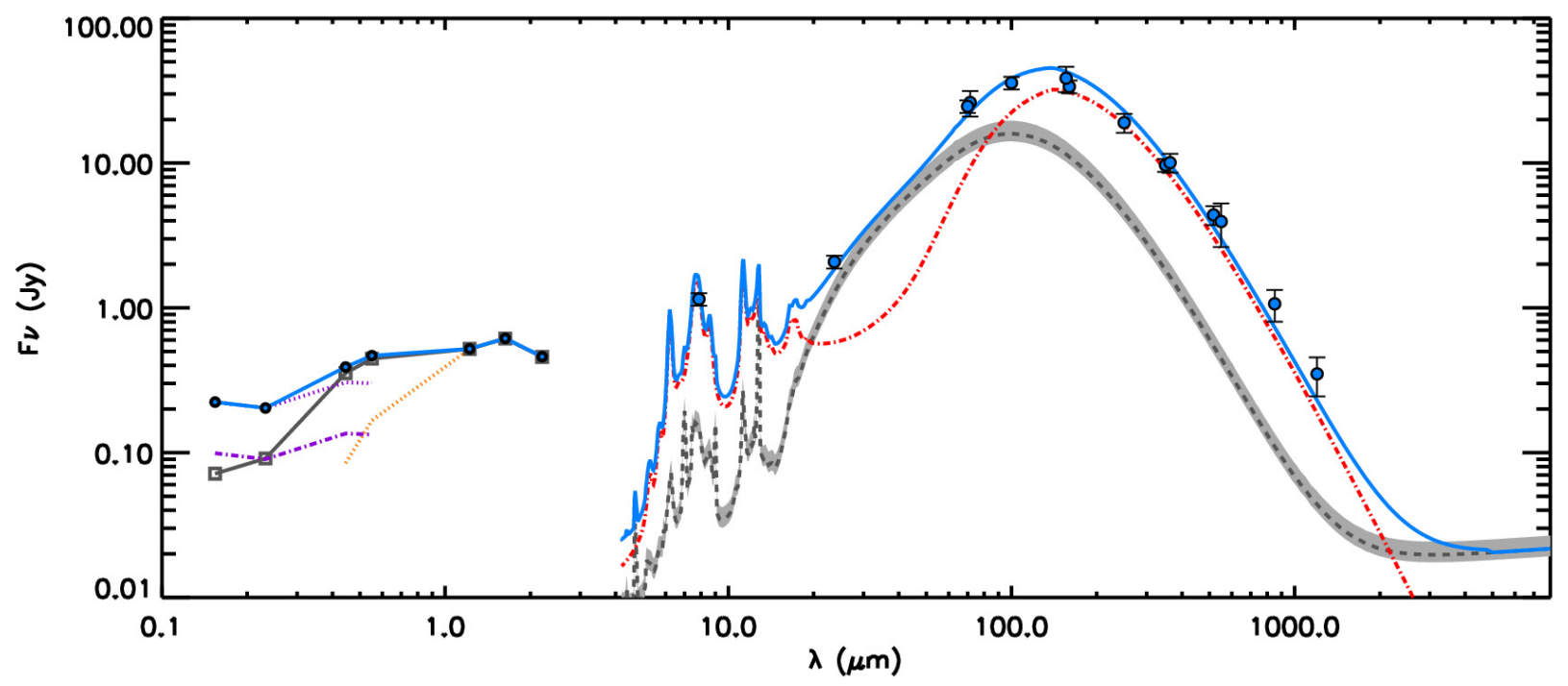

Fig. 7. SED for the total emission of NGC 4214. In the MIR/FIR/submm part we show the best-fit solution for the total emission (solid blue line) obtained as the sum of the best-fit models of the HII regions (grey dashed line) from Figs. 4 and 5 and the best-fit model to the diffuse emission (red dashed-dotted line) from Fig. 6, obtained for $\tau_{\mathrm{B}}^{\mathrm{f}}=2.0$ and $S F R=0.098 M_{\odot} \mathrm{yr}^{-1}$. In the UV/optical/NIR part, the grey open squares and grey solid line represent the observational data, and the blue-filled circles and the blue solid line are the intrinsic fluxes derived from deattenuation of the observed data points as described in Sect. 6.3. The orange dotted line corresponds to the intrinsic emission of the old stellar population (see Sect. 5.2 for details). The dotted purple line shows the intrinsic emission from the young stellar population from the UV to the optical wavelength range. The emission of the young stellar population in the $B$ and $V$ band was obtained after subtracting the contribution of the old stellar component (orange dotted line). The purple dashed-dotted line is the scaled emission of the young stellar population by the factor needed to predict the same $S F R$ as derived from the dust SED modelling.

and a total luminosity of the $\mathrm{CO}$ emission of $L_{\mathrm{CO}(2-1)}=1.7 \times$ $10^{7} \mathrm{~K} \mathrm{~km} \mathrm{~s}^{-1} \mathrm{pc}^{2}$ has been measured. With a Galactic CO-to$\mathrm{H}_{2}$ conversion factor (X-factor, adopted here as $\mathrm{N}\left(\mathrm{H}_{2}\right) / I_{\mathrm{CO}}=$ $\left.2 \times 10^{20} \mathrm{~cm}^{-2}\left(\mathrm{~K} \mathrm{~km} \mathrm{~s}^{-1}\right)^{-1}\right)$, and a line ratio of $I_{\mathrm{CO}(2-1)} / I_{\mathrm{CO}(1-0)}=$ 0.7 (Schruba et al. 2012) this gives $M_{\mathrm{H} 2}=7.9 \times 10^{6} M_{\odot}$, about $50 \%$ higher than the value of $M_{\mathrm{H} 2}=5.1 \times 10^{6} M_{\odot}$ derived from interferometric observations by Walter et al. (2001). Schruba et al. (2012) applied a stacking technique on the HERCULES data in order to detect faint emission and derived a total luminosity of $L_{\mathrm{CO}(2-1)}=3.2 \times 10^{7} \mathrm{~K} \mathrm{~km} \mathrm{~s}^{-1} \mathrm{pc}^{2}$, yielding $M_{\mathrm{H} 2}=1.5 \times$ $10^{7} M_{\odot}$. With this latter value we obtain, taking a helium fraction of 1.36 into account, a total gas mass of $M_{\text {gas }}=5.78 \times 10^{8} M_{\odot}$. The total gas-to-dust mass ratio is then $G_{\text {dust }}=356$.

The Galactic X-factor most likely severely underestimates the total molecular gas mass in NGC 4214. An indication for this is e.g. the very high CII/CO ratio measured in the central region (Cormier et al. 2010), which shows that a large fraction of the $\mathrm{CO}$ is photo-dissociated owing to the high radiation field and low dust shielding. Even though the X-factor has been notoriously difficult to determine, progress has been made in recent years mainly due to the possibility to derive dust masses with an increasingly better precision. The dust mass, together with $\mathrm{HI}$ and $\mathrm{CO}$ measurements and the assumption of a constant gas-to-dust mass ratio, permits to derive the ratio between total molecular gas mass and CO emission. Israel (1997) first used this method and derived for NGC 4214 a 15-30 times higher X-factor than the Galactic value. Leroy et al. (2011) used spatially resolved data for the dust masses, derived from fits to Herschel and Spitzer data, and the gas mass from HI and $\mathrm{CO}$ measurements for a small sample of nearby galaxies (M 31, M 33, LMC, SMC and NGC 6822). They found evidence of a strong increase in the $\mathrm{X}$-factor below metallicities in $12+\log (\mathrm{O} / \mathrm{H})=8.2-8.4$, most likely due to the dissociation of $\mathrm{CO}$ and the creation of extended layers of $\mathrm{CO}$-free $\mathrm{H}_{2}$. Leroy et al. (2011) find for NGC 6822, which has a similar metallicity to NGC $4214(12+\log (\mathrm{O} / \mathrm{H})=8.2)$ an X-factor of four to five times the Galactic value. For the same galaxy, NGC 6822, and a similar method, Gratier et al. (2010) derived a higher X-factor (20 times the Galactic value). Taking this range of estimates into account, we adopt an X-factor ten times the Galactic value as a reasonable estimate and derive, based on $L_{\mathrm{CO}(2-1)}=3.2 \times 10^{7} \mathrm{~K} \mathrm{~km} \mathrm{~s}^{-1} \mathrm{pc}^{2}$ (Schruba et al. 2012), a gasto-dust mass ratio of 469 for this case.

If we assume that the gas-to-dust mass ratio scales linearly with metallicity (which means that the fraction of metals incorporated in the dust is constant) we expect a value of $G_{\text {dust }}$ between 375 (for $Z=0.4 Z_{\odot}$ ) to 500 (for $Z=0.3 Z_{\odot}$ ), based on the solar value of about 150 . Thus, the observed gas-to-dust ratio is very close to the expected value.

\section{Discussion}

The analysis presented in this paper enables us to draw conclusions about the physical properties of the dwarf galaxy under study. The way this can be achieved is by confirming or rejecting the basic ingredients of the models used to fit the data, from the consistency between model predictions and data. Since our models are radiative transfer models, they contain a wealth of information regarding the distribution of stars and dust in galaxies, the clumpiness of the ISM, the dust opacity of the dust clouds, the contributions of the different phases of the ISM, and the optical properties of the dust grains, including the relative abundance of PAH molecules.

Although we can fit the FIR SED of this galaxy, the main discrepancy is that the UV emission is underpredicted with respect to our corresponding predictions for attenuation of UV light. We discuss possible causes for this discrepancy, as well as consequences for the derived physical parameters of this galaxy. We also discuss the underprediction of the $8 \mu \mathrm{m}$ emission in the two central starforming regions, and its implications. 


\subsection{Emission from PAHs at $8 \mu \mathrm{m}$}

We found that the observed emission at $8 \mu \mathrm{m}$ is overpredicted for the diffuse emission, and severely underpredicted for the emission from the HII regions. One reason for this could be, as we discussed in Sect. 3.1, that part of the $8 \mu \mathrm{m}$ flux seen in the apertures, although powered by UV light from the star cluster, is actually likely to originate in the diffuse dust layer beyond the PDR + HII region considered by the Groves et al. (2008) model. Although it is impossible to quantify the importance of this effect, we do not think that it is entirely responsible for the discrepancies, because most of the $8 \mu \mathrm{m}$ emission is clearly associated with the SF regions, as shown by the close correspondence between the $8 \mu \mathrm{m}$ and $\mathrm{H} \alpha$ distribution (e.g. in the shell structure in the NW region).

The model of Popescu et al. (2011) assumes Galactic dust properties and a fraction of PAHs in dust that is appropriate for our Galaxy. The PAH fraction in NGC 4214 might be lower given the lower metallicity (e.g. a lower PAH fraction has been found in the diffuse ISM in the SMC by Sandstrom et al. 2010). If this is the case, we can understand the discrepancy quantitatively.

A possible reason for the excess of $8 \mu \mathrm{m}$ emission in the HII regions may be that the model of Groves et al. (2008) overpredicts the $\mathrm{PAH}$ destruction for this particular object. In the models of Groves et al. (2008) the PAH abundance is fixed to be proportional to the gas metallicity. In addition to this, it includes a parameter that controls the destruction of the PAHs in intense radiation fields. Thus, this model considers PAH destruction and assumes a lower fraction of PAHs in dust in the SF regions.

The observations of Engelbracht et al. (2008) indeed suggest that NGC 4214 might be a galaxy with an unusually high $8 \mu \mathrm{m}$ emission for a given radiation field. They find a good correlation between the equivalent width at $8 \mu \mathrm{m}, \operatorname{EW}(8 \mu \mathrm{m})$, and the ionization parameter for a set of starburst galaxies. The relation has also been confirmed for HII regions in M101 (Gordon et al. 2008). NGC 4214, one of the starburst galaxies in the Engelbracht et al. (2008) sample, deviates significantly from this correlation: it exhibits an $\mathrm{EW}(8 \mu \mathrm{m})$ a factor of three higher than the one corresponding to the ionization parameter. The spectra used in this analysis are not restricted to the SF regions and thus might contain emission from the diffuse medium (Engelbracht et al. 2008 do not quantify this) and therefore we cannot rigorously compare this result to our findings for the SF complexes. However, since the lines from the ionized atoms and the PAH emission come to a large extent from HII regions and their PDRs (see our Tables 1-3) the result of Engelbracht et al. (2008) suggests that the $8 \mu \mathrm{m}$ emission in the SF regions in NGC 4214 might indeed be high.

A final possibility for explaining the high $8 \mu \mathrm{m}$ emission from the SF region is the ratio between neutral and ionized PAHs. The model of Groves et al. (2008) uses templates fitted to the starburst galaxies NGC 4676 and NGC 7252 with a corresponding ratio between neutral and ionized PAHs. This ratio might not be entirely appropriate for the low-metallicity galaxy NGC 4214. Ionized PAHs emit about a factor of ten more energy in the 6-9 $\mu \mathrm{m}$ range than at $11-12 \mu \mathrm{m}$, whereas neutral PAHs emit about the same amount in both ranges (Draine \& Li 2007). Thus, a higher ionized PAH fraction could boost the predicted emission at $8 \mu \mathrm{m}$ at the expense of the emission at 11-12 $\mu \mathrm{m}$. If we assume as an extreme case that the entire emission at $11-12 \mu \mathrm{m}$ is transferred to the $8 \mu \mathrm{m}$ band, we can increase the emission for our model spectrum at $8 \mu \mathrm{m}$ by a factor of $\sim 2$. Thus, we could indeed improve the agreement between data and model, although some discrepancy would remain.

\subsection{The UV emission illuminating the diffuse dust}

In Sect. 6 we found that there is tension between the observed surface brightness of the disk in the submm and the observed flux of the galaxy in the non-ionizing UV in the sense that, although the FIR SED can be fitted, the UV emission is underpredicted by a factor $\sim 2-3$ with respect to our corresponding predictions for attenuation. Here we discuss the plausibility of such an escape fraction and study further possibilities to explain the differences between model and data.

\subsubsection{Escape of unattenuated UV radiation from NGC 4214}

In starburst galaxies, it has been previously observed that a fraction of the UV luminosity can escape from the galaxies without heating the dust, mainly due to the porosity of the ISM created by the energy input of massive stars and SNe, which in the extreme case leads to (super)bubbles and galactic winds. Oey et al. (2007) show that starburst galaxies present a low diffuse $\mathrm{H} \alpha$ emission consistent with an expected escape fraction of ionizing photons of $25 \%$ from the galaxy.

Other studies show that the escape of ionizing radiation (and therefore non-ionizing as well) could be much lower: Leitherer et al. (1995) pointed out that 3\% of the intrinsic LyC photons escape from a set of starburst galaxies; Grimes et al. (2009) find an even lower $(<1 \%)$ fraction for local starburst galaxies; and Siana et al. (2007) find a low escape fraction for low (subsolar) metallicity starburst galaxies $(<8 \%)$.

However, the detection of the escaping photons might be determined by the geometry and orientation of the observations if holes and open channels are the medium that the radiation uses to escape from the galaxy (Clarke \& Oey 2002; Zastrow et al. 2011). Besides, the escape fraction can be affected by the internal properties of galaxies, depending sensitively on the covering factor of clumps, and the density of the clumped and interclumped medium (Fernandez \& Shull 2011).

Conditions that are favourable for an escape are likely to be given in starburst dwarf galaxies, which generally show large bubbles and shell structures (Martin 1998) - similar to what we are seeing in the centre of NGC 4214 - that would make the ISM more porous and prone to letting the UV radiation escape. The simulations of Wise \& Cen (2009) show that an escape fraction as high as $25-80 \%$ can be expected owing to the irregular morphology of the dwarf galaxies with a clumpy ISM. There are observational confirmations of such an escape. Cormier et al. (2012) find evidence of an escape of $80 \%$ of the nonionizing UV-radiation from the low-metallicity Haro 11, and Bergvall et al. (2006) find an escape fraction of the Lyman continuum radiation of $4-10 \%$ in the same object. Zastrow et al. (2011) detected an ionized cone above a shell structure in the starburst galaxy NGC 5253, suggesting that ionizing radiation could be escaping from this galaxy. In the presence of such channels we would expect that not only ionizing, but also non-ionizing radiation escape from the galaxies without being affected by dust.

The geometry of the central SF regions, especially in the case of the NW region, suggests that bubbles have already been created in NGC 4214. We can make an estimate of how much such a process could affect NGC 4214 by assuming that the entire UV luminosity from these regions leaves the galaxy unattenuated. The UV luminosity of the two main HII regions represent $20 \%$ of the total UV luminosity of NGC 4214. 
Therefore, about $20 \%$ of the total UV luminosity possibly does not contribute to the dust heating and should be subtracted when converting into the $S F R^{\prime}$ heating the dust in the disk. This fraction is close to the lower limit of the observed discrepancy and thus might be able to explain a considerable part of it.

\subsubsection{A different geometry of dust and stars in NGC 4214}

In our modelling we have assumed that NGC 4214 is a scaleddown version of the model spiral galaxy used in Popescu et al. (2011). However, the differences in the geometry for the spiral and dwarf galaxies can affect the comparison of the data with the model of Popescu et al. (2011).

The relative scaleheights and scalelengths of the dust and the stars are derived from a sample of edge-on spiral galaxies (Xilouris et al. 1999). However, the geometry of dwarf galaxies is known to be different from that of larger spiral galaxies. In particular, their stellar disks are thicker in comparison to their diameter (e.g. Hunter \& Elmegreen 2006). This is not unexpected because the scaleheight depends on the mass surface density, which determines the gravitational potential in the vertical direction and the velocity dispersion. Both are generally similar in dwarf galaxies and in spiral galaxies, therefore the absolute value of the vertical scaleheight is expected to be similar, and, since they are smaller than spiral galaxies, the ratio between scaleheight and scalelength increases. The gas scaleheight is even higher in absolute terms than in spiral galaxies (Brinks et al. 2002). Thus, we would expect higher ratios between the vertical scaleheights and the radial scalelengths than the one assumed in the model. In NGC 4214 Maíz-Apellániz et al. (1999) estimates the vertical stellar scaleheight to be about 200 pc, i.e. about one fourth to one fifth of the radial scalelength. This is at least a factor of three higher than the ratio assumed in the Popescu et al. (2011) model. However, the effects of a difference in the ratio between scaleheight and scalelength are not expected to be very strong as long as the relative scale ratios between stars and dust are the same.

Another possible, albeit speculative, difference between the model and the real geometry could be a non-central position of the NW and SE SF regions. Since these regions dominate the young SF activity in the galaxy and are very likely responsible for a large fraction of the UV emission, their possible position above or below the galactic disk would have a strong impact on the dust heating, whereas in large galaxies such an asymmetry is unlikely due to the high number of SF regions.

The geometry of the galaxy can also affect the way we are using the attenuation in the disk. The model for attenuation assumes that the starforming complexes are distributed in a thin disk, with a radially decreasing exponential distribution. If instead NGC 4214 had most of the SF occurring in its centre, then the model for attenuation in the UV may overpredict the attenuation if the high local concentration of stars has the effect of breaking up the dust layer.

\subsubsection{An extended dust component}

If the dust is more extended compared to the stellar disk than what is assumed in the model, such an extended dust component will be relatively cold and emit more in the submm. By including such a component we would therefore be able to use a higher value of $S F R^{\prime}$ for the rest of the dust and decrease the discrepancy with the observed UV emission. Furthermore, an extended, cold dust component could explain the missing flux at the long wavelengths. Popescu et al. (2002) have found indications of such an extended dust component with ISO data in dwarf galaxies, however their data lacked the spatial resolution to directly measure its spatial extent. To test whether there are indications of an extended dust component, we derived the radial scalelengths from SPIRE 250, 350, and $500 \mu \mathrm{m}$ bands. The rather irregular distribution of the dust emission did not allow us to define elliptical isophotes at all galactocentric radii, so instead we derived the median values with the isophotal elliptic annuli defined in the g-band image. By carrying out a least-square fit to the radial distribution of these median values, we derived scalelengths of $835 \mathrm{pc}, 920 \mathrm{pc}$, and $1067 \mathrm{pc}$ for SPIRE 250, 350, and $500 \mu \mathrm{m}$, respectively. The increase in the scalelengths from shorter to longer wavelengths is expected because of the decrease in the dust temperature towards the outer parts of the galaxy. The longest wavelength (SPIRE $500 \mu \mathrm{m}$ ) best represents the scalelength of the dust distribution but most likely still underestimates it.

The model of Popescu et al. (2011) adopts a dust scalelength of $1.406 \times h_{\mathrm{s}}$. With $h_{\mathrm{s}}=873 \mathrm{pc}$ this would predict a dust scalelength of $1227 \mathrm{pc}$, which is slightly higher than the measured scalelength at SPIRE $500 \mu \mathrm{m}$ band. Thus, although we cannot exclude the presence of an extended dust component because the dust scalelength can be longer than that at SPIRE $500 \mu \mathrm{m}$ band, we do not find any clear evidence for its presence.

\subsubsection{Different dust properties}

Until now we have mainly discussed different geometrical scenarios for explaining the discrepancies we find in the UV. There is, however, another possibility that the submm mass absorption coefficient of grains, as specified by the Weingartner \& Draine (2001) model and as used in the radiation transfer model of Popescu et al. (2011), underestimates the true mass absorption coefficient of the grains in the submm, leading to lower dust surface densities and lower disk opacity in the UV for the observed submm brightness of the diffuse disk. This is a solution that unfortunately largely degenerates with the assumed geometry of stars and dust. Because of this and the lack of independent constraints on the existence of such a dust model, we cannot draw any conclusion on this issue.

\subsubsection{Physical implications}

In the previous sections we discussed a few possibilities that might have caused the discrepancy in the predicted level of UV emission. Probably the most likely scenario is that dynamical effects associated with a recent burst of SF activity responsible for the central SF complexes has punched holes through the diffuse dust layer, through which the UV photons leaving the SF regions can escape the galaxy without interacting with the diffuse dust. This would be a natural outcome of the non-steady state behaviour of SF activity in dwarf galaxies, in which we may be observing NGC 4214 in a post-starburst phase, where the star clusters are no longer fully cocooned by dust, but on the contrary have evolved to the point where SNe and wind activity have cleared the dust in the ISM above the location of the starburst.

The existence of different grain properties is also plausible, though difficult to distinguish from the other scenarios. This would increase the inferred gas-to-dust mass ratios compared to the radiation transfer model predictions, bringing it even closer to the values expected from the observed metallicity of NGC 4214. Furthermore, dust grains with a higher 
submm emissivity and a flatter frequency dependence would be able to better explain the dust emission at long wavelengths $(850 \mu \mathrm{m})$ where our model presently underpredicts the observations by $34 \%$.

\section{Summary and conclusions}

We have analysed and modelled the dust SED of the nearby starbursting dwarf galaxy NGC 4214. Thanks to its proximity we were able to derive the dust SED separately for the emission from the two major massive SF regions and for the diffuse dust. For the first time this analysis was done from the perspective of a self-consistent radiation transfer calculation constrained by the observed SEDs of spatially separated components on resolved maps. In making predictions for the UV/optical-FIR/submm SEDs of these components this analysis quantitatively considers the level and colour of UV/optical radiation fields incident on both pc-sized dusty structures in the SF complexes and on dust grains distributed on kpc scales in the diffuse ISM, illuminated by a combination of UV light escaping from the SF regions and the ambient optical radiation fields, as constrained by UV/optical photometry of the galaxy. The overall analysis was done using the model predictions and the formalism from Popescu et al. (2011), while for the detailed modelling of the two central SF complexes, we used the model of Groves et al. (2008). The large amount of ancillary data and results from previous studies allowed us to constrain a major part of the input parameters of the models.

We achieved good agreement between data and models, both for the diffuse dust emission and the dust in HII + PDR regions. For the comparison with the dust emission from HII + PDR regions we could constrain basically all input parameters (metallicity, age of star cluster, external pressure, heating capacity, and covering factor) from observations, with the exception of the gas column density of the PDR. We achieved satisfactory fits for both SF regions with the exception of the $8 \mu \mathrm{m}$ data points. Possible reasons for this discrepancy are that the model assumptions (PAH abundance and destruction) are not completely adequate for the case of NGC 4214 or that NGC 4214 has an unusually high emission at $8 \mu \mathrm{m}$ for its metallicity and radiation field, which is supported by other studies (Engelbracht et al. 2008).

We could fit the diffuse dust SED satisfactorily, but we inferred that the UV emission was severely underpredicted with respect to the observed, deattenuated diffuse UV flux. We discussed different explanations for this discrepancy (escape of UV emission, geometrical effects, a very extended dust disk and different dust properties). The most plausible one is that part (40-65\%) of the UV radiation that escapes from HII + PDR regions leaves the galaxy unattenuated and is thus not participating in the heating of the diffuse dust, most likely due to a porous ISM.

We derived a global gas-to-dust mass ratio of 350-470, close to the value expected from the metallicity of NGC 4214 ( $Z=$ $0.3-0.4 Z_{\odot}$ ) of $375-500$.

In summary, this is the first time a full radiation transfer analysis constrained by the observed SEDs of spatially separated components has been done for a dwarf galaxy. Corresponding radiation transfer studies of resolved star-forming dwarf galaxies but with different inclinations and evolutionary states to NGC 4214 may allow the alternative physical scenarios shaping the panchromatic SEDs of these systems to be distinguished.

Acknowledgements. We thank the referee, F. Galliano, whose comments helped to improve the clarity of the manuscript, Joerg Fischera for insightful discussions about the ISM, and Médéric Boquien for help with the Herschel data.
This work has been supported by the research projects AYA2007-67625-C0202 and AYA2011-24728 from the Spanish Ministerio de Ciencia y Educación and the Junta de Andalucía (Spain) grants FQM108. I.H. was supported by a $\mathrm{PhD}$ grant from the Spanish Ministerio de Ciencia y Educación (BES2008-008108). Part of this research has been supported by the ERG HERSFR from the EC. This work is based on observations with the Instituto de Radioastronomía Milimétrica IRAM $30 \mathrm{~m}$. This research made use of the NASA/IPAC Extragalactic Database (NED), which is operated by the Jet Propulsion Laboratory, California Institute of Technology, under contract with the National Aeronautics and Space Administration. We also acknowledge the use of the HyperLeda database (http://leda.univ-lyon1.fr). This research made use of Montage, funded by the National Aeronautics and Space Administration's Earth Science Technology Office, Computational Technnologies Project, under Cooperative Agreement Number NCC5-626 between NASA and the California Institute of Technology. The code is maintained by the NASA/IPAC Infrared Science Archive.

\section{References}

Asplund, M., Grevesse, N., \& Sauval, A. J. 2005, in Cosmic Abundances as Records of Stellar Evolution and Nucleosynthesis, eds. T. G. Barnes, III, \& F. N. Bash, ASP Conf. Ser., 336, 25

Baes, M., Fritz, J., Gadotti, D. A., et al. 2010, A\&A, 518, L39

Baes, M., Verstappen, J., De Looze, I., et al. 2011, ApJS, 196, 22

Bendo, G. J., Dale, D. A., Draine, B. T., et al. 2006, ApJ, 652, 283

Bergvall, N., Zackrisson, E., Andersson, B.-G., et al. 2006, A\&A, 448, 513

Bianchi, S. 2008, A\&A, 490, 461

Bot, C., Ysard, N., Paradis, D., et al. 2010, A\&A, 523, A20

Brinks, E., Walter, F., \& Ott, J. 2002, in Disks of Galaxies: Kinematics, Dynamics and Peturbations, eds. E. Athanassoula, A. Bosma, \& R. Mujica, ASP Conf. Ser., 275, 57

Clarke, C., \& Oey, M. S. 2002, MNRAS, 337, 1299

Condon, J. J. 1992, ARA\&A, 30, 575

Cormier, D., Madden, S. C., Hony, S., et al. 2010, A\&A, 518, L57

Cormier, D., Lebouteiller, V., Madden, S. C., et al. 2012, A\&A, 548, A20

da Cunha, E., Charlot, S., \& Elbaz, D. 2008, MNRAS, 388, 1595

Dale, D. A., Helou, G., Contursi, A., Silbermann, N. A., \& Kolhatkar, S. 2001, ApJ, 549, 215

Dale, D. A., Cohen, S. A., Johnson, L. C., et al. 2009, ApJ, 703, 517

Dale, D. A., Aniano, G., Engelbracht, C. W., et al. 2012, ApJ, 745, 95

de Vaucouleurs, G., de Vaucouleurs, A., Corwin, Jr., H. G., et al. 1991a, S\&T, 82,621

de Vaucouleurs, G., de Vaucouleurs, A., Corwin, Jr., H. G., et al. 1991b, Third Reference Catalogue of Bright Galaxies. Volume I: Explanations and references. Volume II: Data for galaxies between 0h and 12h. Volume III: Data for galaxies between $12 \mathrm{~h}$ and $24 \mathrm{~h}$.

Draine, B. T., \& Hensley, B. 2012, ApJ, 757, 103

Draine, B. T., \& Li, A. 2007, ApJ, 657, 810

Draine, B. T., Dale, D. A., Bendo, G., et al. 2007, ApJ, 663, 866

Engelbracht, C. W., Blaylock, M., Su, K. Y. L., et al. 2007, PASP, 119, 994

Engelbracht, C. W., Rieke, G. H., Gordon, K. D., et al. 2008, ApJ, 678, 804

Fazio, G., \& Pahre, M. 2004, in Spitzer Proposal ID \#69, 69

Fazio, G. G., Hora, J. L., Allen, L. E., et al. 2004, ApJS, 154, 10

Fernandez, E. R., \& Shull, J. M. 2011, ApJ, 731, 20

Galametz, M., Madden, S., Galliano, F., et al. 2009, A\&A, 508, 645

Galametz, M., Madden, S. C., Galliano, F., et al. 2011, A\&A, 532, A56

Galliano, F., Madden, S. C., Jones, A. P., et al. 2003, A\&A, 407, 159

Galliano, F., Madden, S. C., Jones, A. P., Wilson, C. D., \& Bernard, J.-P. 2005, A\&A, 434, 867

Galliano, F., Dwek, E., \& Chanial, P. 2008, ApJ, 672, 214

Gordon, K. D., Engelbracht, C. W., Rieke, G. H., et al. 2008, ApJ, 682, 336

Gratier, P., Braine, J., Rodriguez-Fernandez, N. J., et al. 2010, A\&A, 512, A68

Grimes, J. P., Heckman, T., Aloisi, A., et al. 2009, ApJS, 181, 272

Grossi, M., Hunt, L. K., Madden, S., et al. 2010, A\&A, 518, L52

Groves, B., Dopita, M. A., Sutherland, R. S., et al. 2008, ApJS, 176, 438

Helou, G., Roussel, H., Appleton, P., et al. 2004, ApJS, 154, 253

Hunter, D. A., \& Elmegreen, B. G. 2006, ApJS, 162, 49

Israel, F. P. 1997, A\&A, 328, 471

Israel, F. P., Wall, W. F., Raban, D., et al. 2010, A\&A, 519, A67

Jarrett, T. H., Chester, T., Cutri, R., Schneider, S. E., \& Huchra, J. P. 2003, AJ, 125,525

Karachentsev, I. D., Karachentseva, V. E., Huchtmeier, W. K., \& Makarov, D. I. 2004, AJ, 127, 2031

Kepley, A. A., Zweibel, E. G., Wilcots, E. M., Johnson, K. E., \& Robishaw, T. 2011, ApJ, 736, 139

Kobulnicky, H. A., \& Skillman, E. D. 1996, ApJ, 471, 211

Leitherer, C., Ferguson, H. C., Heckman, T. M., \& Lowenthal, J. D. 1995, ApJ, 454, L19 
Leitherer, C., Vacca, W. D., Conti, P. S., et al. 1996, ApJ, 465, 717

Leroy, A. K., Walter, F., Bigiel, F., et al. 2009, AJ, 137, 4670

Leroy, A. K., Bolatto, A., Gordon, K., et al. 2011, ApJ, 737, 12

Lisenfeld, U., Israel, F. P., Stil, J. M., \& Sievers, A. 2002, A\&A, 382, 860

MacKenty, J. W., Maíz-Apellániz, J., Pickens, C. E., Norman, C. A., \& Walborn,

N. R. 2000, AJ, 120, 3007

MacLachlan, J. M., Matthews, L. D., Wood, K., \& Gallagher, J. S. 2011, ApJ, 741,6

Maíz-Apellániz, J. 2000, PASP, 112, 1138

Maíz-Apellániz, J., Mas-Hesse, J. M., Munoz-Tunon, C., Vilchez, J. M., \& Castaneda, H. O. 1998, A\&A, 329, 409

Maíz-Apellániz, J., Muñoz-Tuñón, C., Tenorio-Tagle, G., \& Mas-Hesse, J. M. 1999, A\&A, 343, 64

Maíz-Apellániz, J., Cieza, L. \& MacKenty, J. W. 2002, AJ, 123, 1307

Martin, C. L. 1998, ApJ, 506, 222

McIntyre, V. J. 1998, PASA, 15, 157

Melisse, J. P. M., \& Israel, F. P. 1994, A\&A, 285, 51

Meny, C., Gromov, V., Boudet, N., et al. 2007, A\&A, 468, 171

Misiriotis, A., Popescu, C. C., Tuffs, R., \& Kylafis, N. D. 2001, A\&A, 372, 775

Natale, G., Tuffs, R. J., Xu, C. K., et al. 2010, ApJ, 725, 955

Oey, M. S., Meurer, G. R., Yelda, S., et al. 2007, ApJ, 661, 801

Planck Collaboration 2011a, A\&A, 536, A17

Planck Collaboration 2011b, A\&A, 536, A7

Planck Collaboration 2011c, VizieR Online Data Catalog, 8088, 0

Popescu, C. C., Misiriotis, A., Kylafis, N. D., Tuffs, R. J., \& Fischera, J. 2000, A\&A, 362, 138

Popescu, C. C., Tuffs, R. J., Völk, H. J., Pierini, D., \& Madore, B. F. 2002, ApJ, 567,221
Popescu, C. C., Tuffs, R. J., Kylafis, N. D., \& Madore, B. F. 2004, A\&A, 414, 45 Popescu, C. C., Tuffs, R. J., Dopita, M. A., et al. 2011, A\&A, 527, A109 Reach, W. T., Dwek, E., Fixsen, D. J., et al. 1995, ApJ, 451, 188 Reach, W. T., Megeath, S. T., Cohen, M., et al. 2005, PASP, 117, 978 Rieke, G. H., Young, E. T., Engelbracht, C. W., et al. 2004, ApJS, 154, 25 Roussel, H. 2012, ArXiv e-prints

Sandstrom, K. M., Bolatto, A. D., Draine, B. T., Bot, C., \& Stanimirović, S. 2010, ApJ, 715, 701

Schruba, A. Leroy, A. K., Walter, F., et al. 2012, AJ, 143, 138

Siana, B., Teplitz, H. I., Colbert, J., et al. 2007, ApJ, 668, 62

Siebenmorgen, R., \& Krügel, E. 2007, A\&A, 461, 445

Silva, L., Granato, G. L., Bressan, A., \& Danese, L. 1998, ApJ, 509, 103 Smith, L. J., Norris, R. P. F., \& Crowther, P. A. 2002, MNRAS, 337, 1309 Swinyard, B. M., Ade, P., Baluteau, J.-P., et al. 2010, A\&A, 518, L4

Tuffs, R. J., Popescu, C. C., Völk, H. J., Kylafis, N. D., \& Dopita, M. A. 2004, A\&A, 419, 821

Úbeda, L., Maíz-Apellániz, J., \& MacKenty, J. W. 2007, AJ, 133, 932

Walter, F., \& Brinks, E. 1999, AJ, 118, 273

Walter, F., Taylor, C. L., Hüttemeister, S., Scoville, N., \& McIntyre, V. 2001, AJ, 121,727

Walter, F., Brinks, E., de Blok, W. J. G., et al. 2008, AJ, 136, 2563

Weingartner, J. C., \& Draine, B. T. 2001, ApJ, 548, 296

Williams, B. F., Dalcanton, J. J., Gilbert, K. M., et al. 2011, ApJ, 735, 22

Wise, J. H., \& Cen, R. 2009, ApJ, 693, 984

Xilouris, E. M., Byun, Y. I., Kylafis, N. D., Paleologou, E. V., \& Papamastorakis, J. 1999, A\&A, 344, 868

Zastrow, J., Oey, M. S., Veilleux, S., McDonald, M., \& Martin, C. L. 2011, ApJ, 741, L17 